\title{
Lattices of choice functions and consensus problems
}

\author{
Bernard Monjardet * $\quad$ Vololonirina Raderanirina $^{\dagger}$
}

\begin{abstract}
In this paper we consider the three classes of choice functions satisfying the three significant axioms called heredity $(\mathrm{H})$, concordance $(\mathrm{C})$ and outcast $(\mathrm{O})$. We show that the set of choice functions satisfying any one of these axioms is a lattice, and we study the properties of these lattices. The lattice of choice functions satisfying $(\mathrm{H})$ is distributive, whereas the lattice of choice functions verifying $(\mathrm{C})$ is atomistic and lower bounded, and so has many properties. On the contrary, the lattice of choice functions satisfying $(\mathrm{O})$ is not even ranked. Then using results of the axiomatic and metric latticial theories of consensus as well as the properties of our three lattices of choice functions, we get results to aggregate profiles of such choice functions into one (or several) collective choice function(s).
\end{abstract}

Key-words: Choice function, lattice, concordance, heredity, outcast, Sperner family, median, consensus.

Title running: Lattices of choice functions

Proofs send to Monjardet B

JEL classification numbers: D71

AMS classification numbers: 91 B14

${ }^{*}$ CERMSEM, Université Paris 1, 106-112 boulevard de l'Hopital, 75647 Paris Cedex 13, France. Email : monjarde@univ-paris1.fr

${ }^{\dagger}$ CERMSEM, Université Paris 1, 106-112 boulevard de l'Hopital, 75647 Paris Cedex 13, France. Email : raderani@aol.com 


\section{Introduction}

Arrow's theorem has fostered a lot of work on the problem to aggregate individual preferences into a collective preference or more generally into a collective choice function. Still more generally the "russian school" (see e.g. [1], [2] or [3]) has considered the problem to aggregate individual choice functions into a collective choice function. In their work they emphasize the role of three axioms on choice functions, namely the heredity (or $\alpha$ or Chernoff or heritage) axiom $(\mathrm{H})$, the concordance (or $\gamma$ or expansion) axiom $(\mathrm{C})$ and the Outcast (or Nash) axiom (O). Indeed, the combination of these axioms gives significant classes of choice functions. For instance, a choice function is "classically" rational (respectively rationalizable by a partial order, or path-independent) if and only if it satisfies axioms $(\mathrm{H})$ and $(\mathrm{C})$ (respectively axioms $(\mathrm{H}),(\mathrm{C})$ and $(\mathrm{O})$, or axioms $(\mathrm{H})$ and $(\mathrm{O}))$. Moreover, one can describe rational -in an extended sense- choice mechanisms, inducing choice functions statisfying each of these three axioms.

In this paper we study the order structure of the sets of choice functions satisfying each of these axioms. Indeed, these sets are always partially ordered by the point-wise order between functions. Moreover, we shall see that they are always lattices, with join and meet operations which can be or not the union and intersection operations.

The interest to specify such structures is in particular linked to consensus problems. Consider for instance individuals using choice mechanisms which induce choice functions satisfying axiom $(\mathrm{O})$. Assume that one takes as consensus the unanimity rule $(x$ is chosen in a set if it is chosen by all the individuals). This amounts to considering the choice function as the intersection of the individual choice functions. But this choice function does not necessarily satisfy axiom $(\mathrm{O})$. In fact, according to a result in [1], it can be a completely arbitrary choice function. Nevertheless, since the set of choice functions is a lattice, one can use the unanimity rule in this lattice (the intersection operation is replaced by the meet operation) and so to get a collective choice function having the same level of rationality as that the individual choice functions have.

Moreover, there exist latticial theories of consensus (see e.g. [6], [11], [15], [17]) which can be applied whenever one aggregates elements of a lattice $L$. In fact, there are two main kinds of approaches and results. The first one is the classical axiomatic approach. The consensus functions (i.e. the functions $L^{n} \mapsto L$ ) must satisfy some "reasonable" axioms and the theory determines the functions satisfying such conditions. The results depend not only on the axioms considered but also on the properties of the lattice and in particular on the properties of dependence relations defined on the sets of its irreducible elements. The second one is the metric approach (a generalization of Kemeny's rule). The consensus element is taken as a "closest" element (relatively to a metric defined on the lattice) to the elements to aggregate. This approach has been particularly developed in the case where the consensus element is a "median" element, a case where one can characterize axiomatically the corresponding "median procedure".

Since our sets of choice functions are lattices one can use the results obtained on the structure of these lattices and those of the latticial theories of consensus to get results on the aggregation problem for such choice functions. This is the process followed in this paper. Then it should be clear that its more original part consists to determine the properties of our lattices of choice functions, since once time these properties are known one has just to apply the relevant general results of the latticial theories. It is also the reason explaining why we don't intend to give all the results which could be obtained by this approach. We will simply give interesting results illustrating this process and we will compare them to some previous results.

In Section 2 we begin by recalling the main notions and results of lattice theory which we need (for some elementary facts not recalled, see e.g. [7], or [8]). In particular we shall use 
the arrow relations defined on a lattice $L$ and the dependence relation $\delta$ (respectively $\beta$ ) defined on the set of join-irreducible (respectively meet-irreducible) elements of $L$.

Section 3 is devoted to studying the structure of sets of choice functions ordered by the point-wise order between functions. We begin by noting that the set of all choice functions on a set $S$ is a Boolean lattice. Its atoms (respectively its coatoms) are the choice functions $c_{A, x}$ (respectively $c^{A, x}$ ) where the choice on a set $X$ is always empty (respectively always equal to $X$ ) except for a set A where the choice is an element $x$ of $A$ (respectively $A-\{x\}$ ). The lattice of choice functions satisfying axiom $(\mathrm{H})$ has a nice structure since it is a distributive lattice with intersection and union as meet and join operations. We determine its meetand join-irreducible elements. Then we study the lattice of choice functions satisying axiom (C). We show that it is atomistic (with atoms the choice functions $c_{A, x}$ ) and we display its meet-irreducible elements. The study of the dependence relation $\delta$ of this lattice allows us to prove that it is lower bounded. Since it is also atomistic, it has many other properties; for instance, it is lower locally distributive and then lower semi-modular and ranked. Finally, we study the much more complex lattice of choice functions satisying axiom $(\mathrm{O})$. Indeed this lattice is coatomistic (with coatoms the choice functions $c^{A, x}$ ) but it is not even ranked. Moreover, its join-irreducible elements seem very difficult to characterize. Nevertheless, we exhibit a class of such join-irreducible elements. The decomposition into "bouquets" associated to a choice function satisying $(\mathrm{O})$ is the tool used to obtain these results.

In Section 4 we begin by recalling some results of the latticial theory of consensus. Then we apply some of these results and the results of the previous Section for the consensus of choice functions satisfying our axioms. According to the structure of the corresponding lattice the same type of axioms asked for the consensus functions can determine a broad class of such functions, those associated to the families of sets called simple games (or federations) or only a restricted class like the class of "oligarchic" or "co-oligarchic" consensus functions. Concerning the metric approach, the median choice function(s) of a profile can be easy or difficult to get according to the structure of the corresponding lattice.

In our conclusion we compare our results with those obtained by the "russian school". In fact there are some identical results, some results leading to the same consensus functions but with different axioms and some different results. We end this paper by pointing out some generalizations of the process followed in this paper and some open problems.

\section{Notations and preliminaries}

General information on lattice theory may for instance be found in Davey and Priestley's book ([8]). Throughout this paper all lattices (or posets) are assumed to be finite. So "lattice" means finite lattice. The following definitions, properties and notations will be particularly useful in the sequel.

Definition $1 A$ poset $(L, \leq)$ is a set $L$ endowed with a partial order $\leq$.

We will often denote it only by $L$.

Definition 2 1. A poset $L$ is a join semilattice if any two arbitrary elements $x$ and $y$ of $L$ have a join (i.e. a least common upper bound) denoted by $x \vee y$.

2. A poset $L$ is a meet semilattice if any two arbitrary elements $x$ and $y$ of $L$ have a meet (i.e. a greatest common lower bound) denoted by $x \wedge y$.

3. A lattice is a poset that is both a meet and a join semilattice. 
A lattice can be denoted by $(L, \leq, \vee, \wedge),(L, \vee, \wedge)$, or simply by $L$. Every subset $A$ of a (finite) lattice $L$ has a join denoted by $\bigvee A$ and a meet denoted by $\wedge A$. We denote by $1_{L}$ or simply by 1 (respectively by $0_{L}$ or simply by 0 ) the greatest element (respectively the least element) of $L$.

Definition 3 1. A subset $S$ of a lattice $(L, \vee, \wedge)$ is a sub join semilattice (or sub$\vee$-semilattice) of $L$ if the join of two arbitrary elements of $S$ belong to $S$.

2. A subset $S$ of a lattice $(L, \vee, \wedge)$ is a sub meet semilattice (or sub- $\wedge$-semilattice) of $L$ if the meet of two arbitrary elements of $S$ belong to $S$.

3. A subset $S$ of a lattice $(L, \vee, \wedge)$ is a sublattice of $L$ if $S$ is both a sub meet semilattice and a sub join semilattice.

Example 1 For any two elements $x, y$ of a lattice $L$ such that $x \leq y$, the interval denoted by $[x, y]$ is the set $[x, y]=\{z \in L: x \leq z \leq y\}$. An interval of $L$ is a sublattice of $L$.

The following well-known ([7]) result will be useful:

Lemma 1 A meet semilattice (respectively a join semilattice) having a greatest element (respectively a least element) is a lattice.

Definition 4 The cover relation on a poset $L$ is the binary relation defined on $L$ by $x \prec y$ if there exists no $z \in L$ such that $x<z<y$.

If $x \prec y$ we say that $y$ covers $x$ or that $x$ is covered by $y$. A poset can be vizualized by its (Hasse) diagram: an element is represented by a point $p_{x}$ of the plane. If $y<x$ then $p_{y}$ is under $p_{x}$ and $p_{y}$ is linked to $p_{x}$ if and only if $y \prec x$. For instance FIG.1 shows the diagram of a lattice $L=\{0, a, b, k, e, f, g, h, 1\}$.

Definition 5 1. An element $j$ of a lattice $L$ is join-irreducible if for $A \subseteq L, j=\bigvee A$ implies $j \in A$, or equivalently if $j$ covers exactly one element of $L$.

2. An element $m$ of a lattice $L$ is meet-irreducible if for $A \subseteq L, m=\bigwedge A$ implies $m \in A$, or equivalently if $m$ is covered by exactly one element of $L$.

3. An atom of $L$ is a (join-irreducible) element of $L$ covering the least element $0_{L}$.

4. A coatom of $L$ is a (meet-irreducible) element of $L$ covered by the greatest element $1_{L}$.

The set of all join-irreducible elements of $L$ is denoted by $J_{L}$ or simply $J$. The set of all meetirreducible elements of $L$ is denoted by $M_{L}$ or simply $M$. The join-irreducible (respectively meet-irreducible) elements allow to get all the elements of $L$ by the formula $x=\bigvee\{j \in J$ : $j \leq x\}$ (respectively $x=\bigwedge\{m \in M: x \leq m\}$ ).

Example 2 In the lattice $L$ of FIG.1, $J_{L}=\{a, b, f, k\}$ and $M_{L}=\{f, g, h, k\} ; L$ has two atoms $a$ and $b$, and two coatoms $g$ and $h$. 


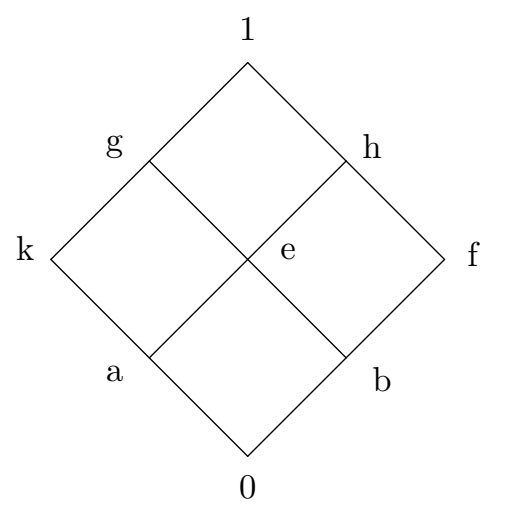

FIG.1 : Lattice $L=\{0, a, b, e, f, g, h, k, 1\}$

An element $x$ of a poset $(L, \leq)$ is maximal if $x \leq y$ implies $x=y$.

Definition 6 Let $L$ be a lattice and $x$ and $y$ be two elements of $L$. The arrow relations are the binary relations defined on $L$ by:

- $x \uparrow y$ if $y$ is maximal in $\{t \in L: x \not \leq t\}$.

- $x \downarrow y$ if $x$ is minimal in $\{t \in L: t \not \leq y\}$.

- $x \uparrow y$ if $x \uparrow y$ and $x \downarrow y$.

It is well known that $x \downarrow y$ (respectively $x \uparrow y$ ) implies $x \in J_{L}$ (respectively $y \in M_{L}$ ). Moreover $J_{L}=\left\{j \in L: \exists m \in M_{L}\right.$ such that $\left.j \downarrow m\right\}$ and $M_{L}=\left\{m \in L: \exists j \in J_{L}\right.$ such that $j \uparrow m\}$. Henceforth we will consider that the dependence relations are defined between the sets $J_{L}$ and $M_{L}: j \uparrow m$ or $j \downarrow m$.

Definition $7 \quad$ 1. The dependence relation $\delta$ on the set $J_{L}$ of join-irreducible elements of a lattice $L$ is defined by : for all $j, j^{\prime} \in J, j \delta j^{\prime}$ if $j=j^{\prime}$ or if there exists a meetirreducible element $m$ of $L$ such that $j \uparrow m$ and $j^{\prime} \not \leq m$ (see [23]).

2. The dual dependence relation $\beta$ is defined on $M_{L}$ by: for all $m, m^{\prime} \in M, m \beta m^{\prime}$ if there exists $j$ in $J_{L}$ satisfying $j \downarrow m$ and $j \not \leq m^{\prime}$.

The arrow and dependence relations of a lattice allow to give useful characterizations of classes of lattices (see for instance Definition 9.8 below).

Definition $8 A$ chain of a lattice $L$ is a subset $A$ of $L$ such that two arbitrary elements of $A$ are always comparable (i.e. $x, y \in A$ implies that $x \leq y$ or $y \leq x$ ). The length of a chain of cardinality $l+1$ is $l$. The length $l(L)$ of a lattice $L$ is the length of a longest chain in $L$.

Example 3 In FIG.1, $l(L)=4$, the length of the chain $0 \prec a \prec k \prec g \prec 1$. 
Lattices of choice functions belong to some classes of lattices that we define below.

Definition 9 1. A lattice $L$ is distributive if it satisfies the distributive laws: for all $x, y, z \in L,(x \vee y) \wedge z=(x \wedge z) \vee(y \wedge z)($ or equivalently $(x \wedge y) \vee z=(x \vee z) \wedge(y \wedge z))$.

2. A lattice $L$ is atomistic if each element $x$ in $L$ is a join of atoms of $L$ (i.e. if $J_{L}$ is the set of all atoms of $L$ ).

3. $L$ is coatomistic if $M_{L}$ is the set of coatoms of $L$.

4. A lattice $L$ is Boolean if it is distributive and atomistic.

5. A lattice $L$ is lower semi-modular if for every $x, y \in L, x \prec x \vee y$ implies $x \wedge y \prec y$. Dually, a lattice $L$ is upper semi-modular if for every $x, y \in L, x \wedge y \prec x$ implies $y \prec x \vee y$.

6. A lattice $L$ is lower locally distributive if its length equals the number of its joinirreducible elements: $l(L)=\left|J_{L}\right|^{1}$.

7. A lattice $L$ is ranked if for any $x$ in $L$ all maximal chains between 0 and $x$ have the same length (see Definition 8) denoted by $r(x)$ and called the rank of $x$ in $L$. Equivalently $L$ is ranked if there exists a map $r$ from $L$ into the set $I N$ of integers such that $x \prec y$ implies $r(y)=r(x)+1$.

8. An atomistic lattice $L$ is lower bounded if its dependence relation $\delta$ defined on $J_{L}$ is acyclic (i.e if there does not exist $j_{1}, j_{2}, \ldots, j_{p}$ distinct elements of $J_{L}(p>1)$ with $\left.j_{1} \delta j_{2}, \ldots, j_{p-1} \delta j_{p}, j_{p} \delta j_{1}\right)^{2}$.

The following result gives some well-known relations between the classes of lattices defined above:

Lemma 2 A Boolean lattice is distributive, a distributive lattice is lower locally distributive, a lower locally distributive lattice is lower semi-modular and a lower semi-modular lattice is ranked.

An atomistic lower bounded lattice is lower locally distributive (and so lower semi-modular and ranked).

Example 4 The lattice of FIG.1 is a distributive, not Boolean lattice.

An example of Boolean lattice is the set of all subsets of a set $S$ (ordered by set inclusion). This lattice has $s=|S|$ atoms and will be denoted by $2^{S}$ or $\underline{2}^{s}$. Since any (finite) Boolean lattice is isomorphic to such a lattice of subsets, a Boolean lattice with $s$ atoms will be also denoted by $2^{s}$.

Remark 1 Note that when $L$ is atomistic (respectively coatomistic), $j \uparrow m$ (respectively $j \downarrow m)$ if and only if $j \uparrow m$.

N.B.: The symbol + (respectively - ) denotes the union of disjoint sets (respectively the difference of sets). We will write $X+x$ (respectively $X-x$ ) rather than $X+\{x\}$ (respectively $X-\{x\})$.

\footnotetext{
${ }^{1}$ Such a lattice has many other equivalent definitions and is also called meet-distributive (see e.g. [22]).

${ }^{2}$ The notion of lower bounded lattice is defined more generally, but here we need to consider only atomistic lower bounded lattices.
} 


\section{$3 \quad$ Lattices of choice functions}

In this Section we deal with lattices of choice functions defined on a finite set $S$ of cardinality $s$. We will first consider the lattice of all choice functions, then successively the lattices of all choice functions verifying each one of the axioms $(\mathrm{H}),(\mathrm{C})$ or $(\mathrm{O})$. For more information on these axioms and their significance in the theory of choice functions see for instance [1], $[3],[4],[25]$.

\subsection{The lattice $\mathcal{S}$ of all choice functions}

Let $S$ be a finite set of cardinality $s$.

Definition $10 A$ choice function on $S$ is a map $c$ from $2^{S}$ to $2^{S}$ satisfying $c(A) \subseteq A$, for every $A \subseteq S$.

So, $c$ is a choice function if $0_{\mathcal{S}} \leq c \leq 1_{S}$ where $1_{S}$ is the identity function on $2^{S}: 1_{\mathcal{S}}(A)=A$, for every $A \subseteq S$, and $0_{\mathcal{S}}$ the null function : $0_{\mathcal{S}}(A)=\emptyset$, for every $A \subseteq S$.

We denote by $\mathcal{S}$ or by $\mathcal{S}(s)$ the set of all choice functions defined on $S$.

Definition 11 We define an order relation $\leq$ on $\mathcal{S}$ by: for all $c, c^{\prime} \in \mathcal{S}, c \leq c^{\prime}$ if for every $A \subseteq S, c(A) \subseteq c^{\prime}(A)$.

Then $\mathcal{S}$ is the interval (see Example 1) $\left[0_{\mathcal{S}}, 1_{\mathcal{S}}\right]$ of the lattice of all functions from $2^{S}$ to $2^{S}$ under the point-wise order.

Let $c$ be a choice function on $S$. In order to simplify the notations, $c(A)=\{x\}$ will be denoted by $c(A)=x$. We introduce the following particular choice functions:

Definition 12 For every subset $U \subseteq S$ and every $x \in U$, we set:

$c_{U, x}(X)=x$ if $X=U$ and $c_{U, x}(X)=\emptyset$ if not;

$c^{U, x}(X)=U-x$ if $X=U$ and $c^{U, x}(X)=X$ if not.

The following result is obvious.

Proposition 1 The ordered set $(\mathcal{S}, \leq)$ of all choice functions on $S$ is a Boolean lattice (Definition 9.4) with join and meet operations defined as follow: for all $c, c^{\prime} \in \mathcal{S}$, for every $A \subseteq S,\left(c \vee c^{\prime}\right)(A)=\left(c \cup c^{\prime}\right)(A)=c(A) \cup c^{\prime}(A)$ and $\left(c \wedge c^{\prime}\right)(A)=\left(c \cap c^{\prime}\right)(A)=c(A) \cap c^{\prime}(A)$. $A$ choice function $c$ is an atom (respectively a coatom) (Definition 5.3) of $\mathcal{S}$ if and only if there exist $U \subseteq S$ and $x \in U$ such that $c=c_{U, x}$ (respectively $c=c^{U, x}$ ).

$A$ choice function $c$ is covered (Definition 4) by a choice function $c^{\prime}$ if $c(X)=c^{\prime}(X)$ except for a set $X$ where $c(X)=c^{\prime}(X)-x$ (with $x \in c^{\prime}(X)$ ).

The rank (Definition 9.7) of the choice function $c$ is $r(c)=\sum_{A \subseteq S}|c(A)|$ and the length (Definition 8) of $\mathcal{S}(s)$ is $s 2^{s-1}$.

According to our general conventions the lattice $(\mathcal{S}, \leq, \cup, \cap)$ of all choice functions on $S$ will be denoted by $\mathcal{S}$ or $\mathcal{S}(s)$. Note that since $\mathcal{S}(s)$ is a Boolean lattice with $s 2^{s-1}$ atoms, $|\mathcal{S}(s)|=2^{s 2^{s-1}}$ (see Example 4). 


\subsection{The lattice $\mathcal{H}$ of choice functions verifying $(\mathbf{H})$.}

A choice function $c$ on a finite set $S$ (of cardinality $s$ ) satisfies the heritage axiom $(\mathbf{H})$ (called also heredity axiom, or Chernoff axiom, or $\alpha$ axiom) if:

for all $A, B \subseteq S, A \subseteq B$ implies $c(B) \cap A \subseteq c(A)$.

Taking the point-wise order relation (Definition 11) on the set $\mathcal{H}$ of all such choice functions we have the following result:

Proposition 2 The poset $(\mathcal{H}, \leq)$ of all choice functions on $S$ satisfying $(H)$ has the same least and greatest element that the lattice $\mathcal{S}$ of all choice functions on $S$. It is a distributive lattice (Definition 9.1) and so a ranked lattice (Definition 9.7 and Lemma 2).

\section{Proof}

It is obvious that the choice functions $0_{\mathcal{S}}$ and $1_{\mathcal{S}}$ satisfy $(\mathrm{H})$ and it is easy to check that the intersection and the union of two choice functions satisfying $(\mathrm{H})$ verify $(\mathrm{H})$. So $(\mathcal{H}, \leq)$ is a sublattice of the Boolean lattice $(\mathcal{S}, \leq)$ and so a distributive lattice.

We will denote by $\mathcal{H}$ or $\mathcal{H}(s)$ the lattice of all choice functions on $S$ satisfying (H).

In order to characterize the join-irreducible elements of the lattice $\mathcal{H}$ we introduce another particular class of choice functions.

Definition 13 Let $x \in V \subseteq S$. We define the choice function $c_{[x, V]}$ on $S$ by: $c_{[x, V]}(X)=x$ if $x \in X \subseteq V$, and $c_{[x, V]}(X)=\emptyset$ if not.

We have the following characterization of the join-irreducible elements of the lattice $\mathcal{H}$.

Proposition 3 A choice function c is a join-irreducible element (Definition 5) of the lattice $\mathcal{H}$ if and only if there exist $V \subseteq S$ and $x \in V$ such that $c=c_{[x, V]}$.

\section{Proof}

First we show that $c_{[x, V]} \in \mathcal{H}$. One has $c_{[x, V]}(Y) \neq \emptyset$ if and only if $x \in Y \subseteq V$ and $c_{[x, V]}(Y)=x$. Let $X \subseteq Y \subseteq V$. If $x \in X$ then $c_{[x, V]}(Y) \cap X=x=c_{[x, V]}(X)$ and $c_{[x, V]}(Y) \cap X=\emptyset=c_{[x, V]}(X)$ if not. So for all $X, Y \subseteq S$ such that $X \subseteq Y$, $c_{[x, V]}(Y) \cap X=c_{[x, V]}(X)$ i.e. $c_{[x, V]} \in \mathcal{H}$.

Now we show that $c \in \mathcal{H}$ implies that $c=\bigcup_{V \subseteq S}\left(\bigcup_{x \in c(V)} c_{[x, V]}\right)$. Let $V \subseteq S$ we check that $\bigcup_{V \subseteq S}\left(\bigcup_{x \in c(V)} c_{[x, V]}\right) \leq c$. Indeed, if $X \subseteq V$ one has $\bigcup_{x \in c(V)} c_{[x, V]}(X)=$ $c(V) \cap X \subseteq c(\bar{X})$ (by $(\mathrm{H}))$, and if not $\bigcup_{x \in c(V)} c_{[x, V]}(X)=\emptyset=c(X)$. Conversely it is obvious that $c \leq \bigcup_{V \subseteq S}\left(\bigcup_{x \in c(V)} c_{[x, V]}\right)$, since for every $X \subseteq S, c(X)=\bigcup_{x \in c(X)} c_{[x, X]}(X) \subseteq$ $\bigcup_{V \subseteq S}\left(\bigcup_{x \in c(V)} c_{[x, V]}(X)\right)$.

Since every choice function in $\mathcal{H}$ is union of choice functions $c_{[x, V]}$ we only have to prove that all such choice functions are join-irreducible in $\mathcal{H}$, i.e. cover a unique choice function of $\mathcal{H}$. We set $c_{[x, V]}^{-}(X)=x$ if $x \in X \subset V$, and $c_{[x, V]}^{-}(X)=\emptyset$ if not. One has $c_{[x, V]}^{-}<c_{[x, V]}$ since they are equal except in the case $X=V$, where $c_{[x, V]}^{-}(V)=c_{[x, V]}(V)-x(=\emptyset)$. Moreover $c_{[x, V]}^{-}$verifies (H). Indeed for all $\emptyset \subset X \subseteq Y \subseteq S, c_{[x, V]}^{-}(Y)$ is not empty if and only if 
$x \in Y \subset V$. In such a case, one has $X \subset V$. Then if $x \in X, c_{[x, V]}^{-}(Y) \cap X=x=c_{[x, V]}^{-}(X)$ and if $x \notin X, c_{[x, V]}^{-}(Y) \cap X=\emptyset=c_{[x, V]}^{-}(X)$.

Now assume that $c \in \mathcal{H}$ and $c<c_{[x, V]}$ and show that $c \leq c_{[x, V]}^{-} \cdot c<c_{[x, V]}$ implies that there exists $A \subseteq S$ such that $x \in A \subset V$ and $c(A)=\emptyset$. One has $c(V) \subseteq c_{[x, V]}(V)=x$. If $c(V)=x$, then $x \in c(V) \cap A \nsubseteq c(A)=\emptyset$, a contradiction with $(\mathrm{H})$. Then $c(V)=\emptyset$. So $c \leq c_{[x, V]}^{-}$.

As consequence of this result one gets that the distributive lattice $\mathcal{H}$ (equivalently its set of join-irreducible elements) has a very particular structure expressed in the following corollary. Recall that $|S|=s$.

Corollary 1 1. The poset $J_{\mathcal{H}}$ of join-irreducible elements of the lattice $\mathcal{H}$ of choice functions verifying $(H)$ is the union of $s$ posets isomorphic to the Boolean lattice $\underline{2}^{s-1}$.

2. The length (Definition 8) of $\mathcal{H}$ is $s 2^{s-1}$ and the rank (Definition 9.7) of $c \in \mathcal{H}$ is $r(c)=\sum_{A \subseteq S}|c(A)| . \mathcal{H}$ is a covering sublattice of $\mathcal{S}:$ for $c, c^{\prime} \in \mathcal{H}, c \prec c^{\prime}$ in $\mathcal{H}$ if and only if $c \prec c^{\prime}$ in $\mathcal{S}$.

3. The lattice $\mathcal{H}$ is isomorphic to the direct product ${ }^{3}$ of s bounded free distributive lattices with $s-1$ generators ${ }^{4}$.

4. $|\mathcal{H}|=\left(d_{s-1}\right)^{s}$ where $d_{s-1}$ is the number of Sperner families ${ }^{5}$ on a set of cardinality $s-1$.

\section{Proof}

1- For $x$ fixed element of $S$ there exist $2^{s-1}$ subsets $V$ of $S$ containing $x$. Then one easily cheks that the poset $\left\{c_{[x, V]}, x \in V \subseteq S\right\}$ is isomorphic to the Boolean lattice $\underline{2}^{s-1}$. Since by Proposition 3, $J_{\mathcal{H}}=\left\{c_{[x, V]}, x \in V \subseteq S\right\}, J_{\mathcal{H}}$ is the union of $s$ posets isomorphic to the Boolean lattice $\underline{2}^{s-1}$. In particular $\left|J_{\mathcal{H}}\right|=s 2^{s-1}$.

2-It is well-known that the length of a distributive lattice is the number of its join-irreducible elements. Then $l(\mathcal{H})=s 2^{s-1}$. Since $\mathcal{H} \subseteq \mathcal{S}$ is a ranked lattice having the same length that $\mathcal{S}$, its maximal chains are maximal chains of $\mathcal{S}$. Then $r(c)$ in $\mathcal{H}$ equals $r(c)$ in $\mathcal{S}$, i.e. $\sum_{A \subseteq S}|c(A)|$ (Proposition 1). Let $c, c^{\prime} \in \mathcal{H}$. It is easy to check that $c \prec c^{\prime}$ in $\mathcal{H}$ if and only if in $\mathcal{S}, c^{\prime}=c \vee c_{U, x}$ with $U$ minimal such that $x \in U$ and $x \notin c(U)$, then if and only if $c \prec c^{\prime}$ in $\mathcal{S}$.

3- Since $\mathcal{H}$ is a distributive lattice it is isomorphic to the lattice of all ideals ${ }^{6}$ of $J_{\mathcal{H}}$ (see [7] or $[8])$. On the other hand the lattice of all ideals of the union of posets is isomorphic to the direct product of the lattices of ideals of these posets. Moreover it is well-known (see [7]) that the lattice of ideals of $\underline{2}^{s-1}$ is isomorphic to the bounded free distributive lattice with $s-1$ generators $F D L(s-1)^{*}$. So (by 1 - just above), $\mathcal{H}$ is isomorphic to the direct product of $s$ lattices, all isomorphic to the lattice of ideals of $\underline{2}^{s-1}$, and then to the direct product of $s$ bounded free distributive lattices with $s-1$ generators.

4- It is well known (see [7]) that the cardinality of the bounded free distributive lattice with $s$ generators equals the number of Sperner families on $S$.

\footnotetext{
${ }^{3}$ The direct product of $s$ lattices $\left(L_{1}, \leq_{1}\right), \ldots,\left(L_{s}, \leq_{s}\right)$ is the poset -in fact a lattice- defined on the set of $s$-tuples $\left(x_{1}, \ldots, x_{s}\right)$ of $L_{1} \times \ldots \times L_{s}$ by $\left(x_{1}, \ldots, x_{s}\right) \leq\left(x_{1}^{\prime}, \ldots, x_{s}^{\prime}\right)$ if for every $i=1, \ldots, s$, $x_{i} \leq_{i} x_{i}^{\prime}$.

${ }^{4}$ The bounded free distributive lattice with $s$ generators, denoted by $F D L(s)^{*}$, is obtained by adding a minimum element 0 and a maximum element 1 to the free distributive lattice with $s$ generators (see [7] for the definition of the free distributive lattice).

${ }^{5}$ See Definition 21 below.

${ }^{6}$ An ideal of a poset $(L, \leq)$ is a subset $I$ of $L$ such that $x \in I$ and $y \leq x$ imply $y \in I$.
} 
Example 5 Take $s=2, S=\{1,2\}$. One has $J_{\mathcal{H}(2)}=\left\{c_{[1,1]}, c_{[1,12]}, c_{[2,2]}, c_{[2,12]}\right\}$ and $F D L(1)^{*}=\{0, g, 1\}$ (the free distributive lattice with a unique generator $g$ equals $\{g\}$ ). These posets are represented on FIG.2.

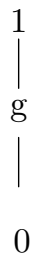

The lattice $F D L(1)^{*}$.

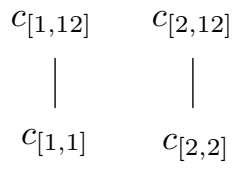

The poset $\left(J_{\mathcal{H}(2)}, \leq\right)$.

FIG.2

One can check on FIG.3 that the lattice $\mathcal{H}(2)$ is isomorphic to the direct product $F D L(1)^{*} \times F D L(1)^{*}$. On this figure a choice function $c$ is denoted by writing for each non-empty subset $A$ of $S$ the subset $c(A)$. A subset $A$ is denoted by the sequence of its elements, and $c(A)$ is denoted by underlining the elements of $A$ which it contains. For instance $c=c_{[1,12]}$ is written $\underline{1}, 2, \underline{12}$ (i.e. $c(\emptyset)=c(2)=\emptyset, c(1)=c(12)=1$ ).

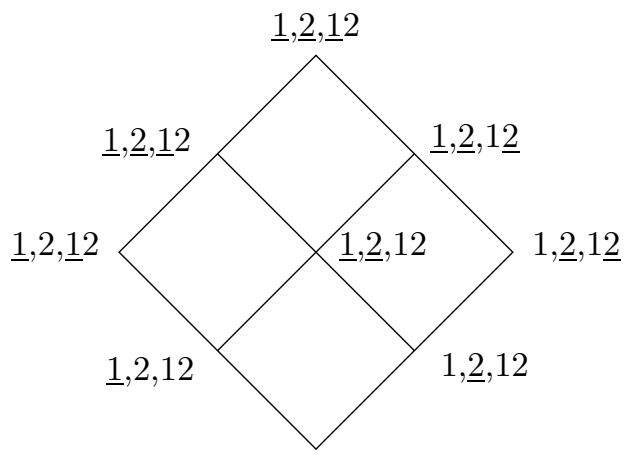

$1,2,12$

FIG.3 : The lattice $\mathcal{H}(2)$ of choice functions satisfying $(\mathrm{H})$.

Remark 2 Since the number of Sperner families on a set of cardinality $s$ (i.e. $\left|F D L(s)^{*}\right|$ ) is known up to $s=7$ (see [26]), then the number of choice functions satisfying $(\mathrm{H})$ is known up to $s=8$. For instance $|\mathcal{H}(3)|=6^{3}$ and $|\mathcal{H}(8)|=(2.414 .682 .040 .998)^{7}$.

Finally, we characterize the meet-irreducible elements of the lattice $\mathcal{H}$. We need to define a particular class of choice functions:

Definition 14 Let $x \in V \subseteq S$. We define the choice function $c^{[x, V]}$ by: $c^{[x, V]}(X)=X-x$ if $V \subseteq X$ and $c^{[x, V]}(X)=X$ if not.

We have the following result: 
Proposition 4 A choice function $c$ is meet-irreducible (Definition 5) in $\mathcal{H}$ if and only if there exist $V \subseteq S$ and $x \in V$ such that $c=c^{[x, V]}$.

\section{Proof}

1) We first show that $c^{[x, V]} \in \mathcal{H}$. Let $\emptyset \subset X \subseteq Y$. If $V \subseteq X$, then $c^{[x, V]}(Y) \cap X=$ $(Y-x) \cap X=X-x=c^{[x, V]}(X)$. If $V \nsubseteq \nsubseteq X, c^{[x, \bar{V}]}(Y) \cap X \subseteq X=c^{[x, V]}(X)$.

2) Now we prove that $c=c^{[x, V]}$ is meet-irreducible, i.e. is covered by a unique element of $\mathcal{H}$. One define the choice function $c_{+}^{[x, V]}$ on $S$ by: $c_{+}^{[x, V]}(X)=X-x$ if $V \subset X$ and $c_{+}^{[x, V]}(X)=X$ if not. One has $c_{+}^{[x, V]}>c^{[x, V]}$ since $c_{+}^{[x, V]}=c^{[x, V]}$ except in the case $X=V$, where $c_{+}^{[x, V]}(V)=c^{[x, V]}(V)+x$. Moreover $c_{+}^{[x, V]} \in \mathcal{H}$. Indeed let $X \subseteq Y \subseteq S$. One has $c_{+}^{[x, V]}(Y) \neq c^{[x, V]}(Y)$ if and only if $Y=V$. Then one has only to consider the case $X \subseteq Y=V$. In this case $c_{+}^{[x, V]}(V) \cap X=V \cap X=X=c_{+}^{[x, V]}(X)$, and so condition (H) is satisfied. Now, let $c \in \mathcal{H}$ with $c^{[x, V]}<c$. We have to prove that $c_{+}^{[x, V]}(V) \leq c$. This is equivalent to prove $c_{+}^{[x, V]}(V)=c(V)=V \cdot c^{[x, V]}<c$ implies that there exists $T$ such that $V \subseteq T \subseteq S$ with $c(T)=T \supset c^{[x, V]}(T)=T-x$. If $V \subset T$ and $c(V)=V-x$, one has $c(T) \cap V=V \nsubseteq c(V)=V-x$, a contradiction with $(\mathrm{H})$. So we have proved that $c(V)=V$. 3) In a distributive lattice the number of join-irreducible elements equals the number of meet-irreducible elements. Since $\left|\left\{c_{[x, V]}, x \in V \subseteq S\right\}\right|=\left|\left\{c^{[x, V]}, x \in V \subseteq S\right\}\right|$ there is no other meet-irreducible element.

\section{The lattice $\mathcal{C}$ of choice functions verifying (C)}

A choice function on a finite set $S$ (of cardinality $s$ ) verifies the concordance axiom (C) (called also expansion axiom or $\gamma$ axiom) if:

$$
\text { for all } A, B \subseteq S, c(A) \cap c(B) \subseteq c(A \cup B) \text {. }
$$

We denote by $\mathcal{C}$ or $\mathcal{C}(s)$ the set of all choice functions verifying $(\mathrm{C})$.

Using the following definition we give other useful characterizations of $(\mathrm{C})$.

Definition 15 Let $A \subseteq S,|A| \geq 2$ and $x \in A$. An $x$-covering of $A$ is a family $\mathcal{R}=\left\{A_{i}\right.$, $i \in I\}$ of subsets of $A$, satisfying $x \in \bigcap_{i \in I} A_{i} \subset \bigcup_{i \in I} A_{i}=A$.

Example 6 The family $\mathcal{R}=\{\{x, y\}, x \in A, y \in A-x\}$ is an $x$-covering of $A \subseteq S$.

We have the following characterizations of $(\mathrm{C})$ :

Proposition 5 Let $c$ be a choice function on $S$. The following properties are equivalent: 1)- $c$ verifies $(C)$,

2)- For all $\left(A_{i}\right)_{i \in I}$ family of subsets of $S, \bigcap_{i \in I} c\left(A_{i}\right) \subseteq c\left(\bigcup_{i \in I} A_{i}\right)$,

3)- For all $A \subseteq S, x \in A-c(A)$ and $\mathcal{R} x$-covering of $A$, there exists $A_{i} \in \mathcal{R}$ such that $x \notin c\left(A_{i}\right)$. 


\section{Proof}

Let $c$ be a choice function on $S$.

$1 \Longleftrightarrow 2$. Indeed 1 implies 2 by recurrence on $|I|$ and the converse is obvious.

$2 \Rightarrow 3$. If not, there exists $A \subseteq S, x \in A-c(A)$ and an $x$-covering $\mathcal{R}=\left(A_{i}\right)_{i \in I}$ of $A$ such that $x \in c\left(A_{i}\right)$ for every $A_{i} \in \mathcal{R}$. So $x \in \bigcap_{i \in I} c\left(A_{i}\right) \not \subset c\left(\bigcup_{i \in I} A_{i}\right)=c(A)$, a contradiction.

$3 \Rightarrow 2$. Let $\mathcal{R}=\left(A_{i}\right)_{i \in I}$ be a family of subsets of $S$ such that $x \in \bigcap_{i \in I} c\left(A_{i}\right)$. One has $x \in A_{i}$ for every $i \in I$. We set $A=\bigcup_{i \in I} A_{i}$ and assume $|A| \geq 2$ (if not the result is obvious). $\mathcal{R}$ is an $x$-covering of $A$. If $x \notin c(A)$ there exists an $A_{i} \in \mathcal{R}$ such that $\left.x \notin c\left(A_{i}\right)\right)$, a contradiction. So $x \in c(A)=c\left(\bigcup_{i \in I} A_{i}\right)$.

It is easy to check that the intersection of two choice functions of $\mathcal{C}$ verifies $(\mathrm{C})$, and it is obvious that the trivial choice functions $0_{\mathcal{S}}$ and $1_{\mathcal{S}}$ satisfy $(\mathrm{C})$. On the other hand the union of two choice functions of $\mathcal{C}$ does not generally verify (C). For instance, with $S=\{1,2,3\}$, $c_{12,1}$ and $c_{13,1}$ (Definition 12) satisfy (C) but not $c_{12,1} \cup c_{13,1}$. But since $\mathcal{C}$ is an $\bigcap$-semilattice containing a greatest element, Lemma 1 gives the following result (where $\leq$ is the point-wise order between choice functions).

Proposition 6 The poset $(\mathcal{C}, \leq)$ of all choice functions on $S$ verifying $(C)$ is a lattice with $0_{\mathcal{S}}$ (respectively $1_{\mathcal{S}}$ ) as least (respectively greatest) element. It is a sub-П-semilattice (Definition 3) of the lattice $\mathcal{S}$ of all choice functions on $S$.

Henceforth the lattice $(\mathcal{C}, \leq)$ is simply denoted by $\mathcal{C}$ or $\mathcal{C}(s)$.

For $|S|=2$, every choice function satisfies $(\mathrm{C})$. Then $\mathcal{C}(2)$ is the Boolean lattice $\mathcal{S}(2)$. This is no longer the case for $|S| \geq 3$. For instance, $|\mathcal{C}(3)|=2744<|\mathcal{S}(3)|=4096$. However we have the following result:

Proposition 7 A choice function $c$ is an atom (Definition 5) of the lattice $\mathcal{C}$ if and only if $c$ is an atom $c_{U, x}$ of the lattice $\mathcal{S}$. Moreover the lattice $\mathcal{C}$ is atomistic (Definition 9.2).

\section{Proof}

Let $c=c_{U, x}$ be an atom of the lattice $\mathcal{S}$, i.e. a choice function such that $c(U)=x$ and $c(X)=\emptyset$, for every $X \neq U$. It is clear that $c$ satisfies $(\mathrm{C})$. Then $c$ is an atom of the lattice $\mathcal{C}$. Since every choice function verifying $(\mathrm{C})$ contains at least an atom of the lattice $\mathcal{S}$ the converse is true. Since the lattice $\mathcal{C}$ is contained in the atomistic lattice $\mathcal{S}$ and has the same atoms it is atomistic too.

Since $\mathcal{C}$ is atomistic its join-irreducible elements (Definition 5) are its atoms determined below. In order to determine its meet-irreducible elements we will use the characterization of the meet-irreducible elements given in Section 2: $c \in M_{\mathcal{C}}$ if and only if there exists a joinirreducible element $j$ of $\mathcal{C}$ such that $j \uparrow c$. Moreover, since $\mathcal{C}$ is atomistic, this characterization is equivalent to write: $c \in M_{\mathcal{C}}$ if and only if there exists an atom $c_{U, x}$ of $\mathcal{C}$ such that $c_{U, x} \uparrow c$ (see Remark 1). We need the following definition, notation and lemmas:

Definition 16 For all $\{x, y\} \subseteq V \subseteq S$, we define the choice function $c^{[x y, V], x}$ by $c^{[x y, V], x}(X)=X-x$ if $\{x, y\} \subseteq X \subseteq V$, and $c^{[x y, V], x}(X)=X$ if not.

Remark 3 Observe that when $V=\{x, y\}$, then $c^{[x y, V], x}=c^{V, x}$ (Definition 12).

Lemma 3 For $\{x, y\} \subseteq V \subseteq S$, one has $c^{[x y, V], x} \in \mathcal{C}$. 


\section{Proof}

Let $A \subseteq S$ such that $t \in A-c^{[x y, V], x}(A)$, i.e $t=x$ and $c^{[x y, V], x}(A)=A-x$. Let $\mathcal{R}=\left(A_{i}\right)_{i \in I}$ be an $x$-covering of $A$. Necessarily, there exist (at least one) $A_{i} \in \mathcal{R}$ such that $\{x, y\} \subseteq$ $A_{i} \subset V$. So, $x \notin c^{[x y, V], x}\left(A_{i}\right)$ and $c^{[x y, V], x} \in \mathcal{C}$ (by Proposition 5.3).

Notation:

Let $V \subseteq S, x \in V$. We set $\mathcal{R}(V, x)=\left\{\mathcal{R}_{1}, \ldots, \mathcal{R}_{m}\right\}$ the set of all $x$-coverings of $V$. When $\mathcal{R} \in \mathcal{R}(V, x), \mathcal{R}=\left\{V_{1}, \ldots, V_{k}\right\}$ and for every $i \in\{1, \ldots, k\}, x \in V_{i}$ and $\bigcup_{i=1}^{k} V_{i}=V$.

Definition 17 Let $V \subseteq S, x \in V$. A family $\mathcal{T}=\left\{T_{1}, \ldots, T_{p}\right\}$ of subsets of $V$ all containing $x$ is a transversal of the set $\mathcal{R}(V, x)$ of all $x$-covering of $V$ if for every $\mathcal{R} \in \mathcal{R}(V, x)$, $\mathcal{T} \cap \mathcal{R} \neq \emptyset$ (i.e., there exists $T_{i} \in \mathcal{R}$ ).

$\mathcal{T}$ is a minimal transversal of $\mathcal{R}(V, x)$ if $\mathcal{T}$ is a transversal of $\mathcal{R}(V, x)$ and for every $T_{i} \in \mathcal{T}, \mathcal{T}-\left\{T_{i}\right\}$ is not a transversal of $\mathcal{R}(V, x)$ (i.e. there exists $\mathcal{R} \in \mathcal{R}(V, x)$ such that $\left.\mathcal{R} \cap\left(\mathcal{T}-\left\{T_{i}\right\}\right)=\emptyset\right)$.

Lemma 4 Let $V \subseteq S, x \in V$. The family $\mathcal{T}$ of subsets of $V$ all containing $x$ is a minimal tranversal of $\mathcal{R}(V, x)$ if and only if $\mathcal{T} \in\{[x y, V], y \in V-x\}$, i.e. if there exists $y \in V-x$ such that $\mathcal{T}=\{T \subseteq S:\{x, y\} \subseteq T \subseteq V\}$.

\section{Proof}

Let $\mathcal{T}=[x y, V]$ for $y \in V-x$ and $\mathcal{R}$ an $x$-covering of $V$. There exists at least $V_{i} \in \mathcal{R}$ such that $y \in V_{i}$. Then $\{x, y\} \subseteq V_{i} \subseteq V$ and $V_{i} \in \mathcal{T}$. So $\mathcal{T}$ is a transversal of $\mathcal{R}(V, x)$. It is a minimal transversal since if $\mathcal{T}^{\prime}=\mathcal{T}-\left\{V_{i}\right\},\{x, y\} \subseteq V_{i} \subseteq V$, the $x$-covering $\mathcal{R}=\left\{V_{i}\right.$, $\left.\left(x+\left(V-V_{i}\right)\right)\right\}$ of $V$ is such that $\mathcal{T}^{\prime} \cap \mathcal{R}=\emptyset$ (indeed $V_{i} \notin \mathcal{T}^{\prime}$ and $y \notin\left[x+\left(V-V_{i}\right)\right]$ implies that $\left.\left[x+\left(V-V_{i}\right)\right] \notin \mathcal{T}^{\prime}\right)$.

$\Longrightarrow$

Assume that there exists a minimal transversal $\mathcal{T}$ of $\mathcal{R}(V, x)$ different from a (minimal) transversal $[x y, V], y \in V-x$. By minimality, for every $y \in V-x,[x y, V] \nsubseteq \mathcal{T}$. Then for every $y \in V-x$ there exists $V(y)$ such that $\{x, y\} \subseteq V(y) \subseteq V$ and $V(y) \notin \mathcal{T}$. But since $\bigcup\{V(y), y \in V-x\}=V, \mathcal{R}=\{V(y), y \in V-x\}$ is an $x$ - covering of $V$. Hence $\mathcal{R} \in \mathcal{R}(V, x)$ and $\mathcal{T} \cap \mathcal{R}=\emptyset$, a contradiction. So $\mathcal{T}=[x y, V]$, for every $y \in V-x$.

We characterize now the relation $\uparrow$, which in this atomistic lattice is equal to the relation $\uparrow$ (Definition 6 and Remark 1).

Proposition 8 Let $c_{U, x}$ be an atom (Definition 5 and Proposition 7) of the lattice $\mathcal{C}$. If $U=\{x\}$ then $\left\{c \in \mathcal{C}: c_{x, x} \uparrow c\right\}=\left\{c^{x, x}\right\}$.

If $|U| \geq 2$ then $\left\{c \in \mathcal{C}: c_{U, x} \uparrow c\right\}=\left\{c^{[x y, U], x}: y \in U-x\right\}$.

\section{Proof}

Let $x \in U \subseteq S$ and $c \in \mathcal{C}$.

If $U=\{x\}$, then $c_{x, x} \uparrow c$ if and only if $c(x)=\emptyset$ and $c$ is maximal with this property. Clearly $c^{x, x}$ is the unique maximal choice function such that $c(x)=\emptyset$. Then $c_{x, x} \uparrow c$ if and only $c=c^{x, x}$.

If $|U| \geq 2$ we first claim that for every $y \in U-x, c_{U, x} \uparrow c^{[x y, U], x}$. Indeed, let $y \in U-x$ and set $c=c^{[x y, U], x}$. One has $x \notin c(U)$ and then $c_{U, x} \not \leq c$. If there exists $c^{\prime} \in \mathcal{C}$ such that $x \notin c^{\prime}(U)$ and $c<c^{\prime}$, then there exists $X$ such that $\{x, y\} \subseteq X \subset U$ with $c(X)=X-x \subset c^{\prime}(X)=X$. But in that case $\{X,(U-X)+x\}$ is an $x$-covering of $U$ with 
$x \in c^{\prime}(X) \cap c^{\prime}((U-X)+x)=c^{\prime}(X) \cap((U-X)+x)$ and $x \notin c^{\prime}(U)$, a contradiction with $c^{\prime} \in \mathcal{C}$.

On the other hand, let $c \in \mathcal{C}$ with $c_{U, x} \uparrow c$. We claim that $c=c^{[x y, U], x}$ for $y \in U-x$. Indeed $c_{U, x} \uparrow c$ implies that $x \notin c(U)$. Hence (by Proposition 5) for every $x$-covering $\mathcal{R}$ of $U$ (i.e. $\mathcal{R} \in \mathcal{R}(U, x)$ ), there exists $U_{\mathcal{R}} \in \mathcal{R}$ such that $x \notin c\left(U_{\mathcal{R}}\right)$. Let us consider the family $\mathcal{T}$ of all these subsets $U_{\mathcal{R}}$, i.e. $\mathcal{T}=\left\{U_{\mathcal{R}}, \mathcal{R} \in \mathcal{R}(U, x)\right\}$. $\mathcal{T}$ is a transversal of $\mathcal{R}(U, x)$. Then $\mathcal{T}$ contains a minimal transversal of $\mathcal{R}(U, x)$, i.e., there exists $y \in U-x$ such that $[x y, U]=\mathcal{T}$ (by Lemma 4). Then for every $X$ such that $\{x, y\} \subseteq X \subseteq U, X \in \mathcal{T}$ and $x \notin c(X)$. Then $c(X) \subseteq X-x=c^{[x y, U], x}(X)$. Hence, $c \leq c^{[x y, U], x}$. But we have proved above that $c_{U, x} \uparrow c^{[x y, U], x}$. Then (by definition of $\uparrow$ ) $c=c^{[x y, U], x}$.

By this proposition and the fact that $M_{\mathcal{C}}=\left\{c \in \mathcal{C}: \exists c_{U, x}\right.$ with $\left.c_{U, x} \uparrow c\right\}$, we get the characterization of the meet-irreductible elements of $\mathcal{C}$ :

Corollary $\mathbf{2}$ Let $c \in \mathcal{C}$. The following properties are equivalent:

1 - $c$ is a meet-irreducible element (Definition 5) of $\mathcal{C}$,

2- There exists $x \in S$ such that $c=c^{x, x}$ (Definition 12) or there exist $U \subseteq S, x, y \in U$ such that $c=c^{[x y, U], x}$ (Definition 16).

Remark 4 One can show that the coatoms (Definition 5 ) of $\mathcal{C}$ are exactly the choice functions $c^{[x y, V], x}$ with $V=\{x, y\}$ and the choice functions $c^{x, x}$, that is to say all the coatoms $c^{V, x}$ of the lattice $\mathcal{S}$ satisfying $|V| \leq 2$ (see [27]).

Now, we determine the dependence relation $\delta$ (Definition 7 ) of the lattice $\mathcal{C}$. We need the following definitions:

Definition 18 Let $\underline{2}^{s}$ be the Boolean lattice (Definition 9.4) with s atoms, and 1 its greatest element.

- The poset $2^{s}-\{1\}$ is called the top truncated Boolean lattice and it is denoted by $\left(\underline{2}^{s}-1\right)$.

- An antichain $A$ of a poset $(L, \leq)$ is a subset of $L$ such that two elements of $A$ are incomparable.

Recall that in a lattice $L$, for $j, j^{\prime} \in J_{L}$, one has $j \delta j^{\prime}$ if and only if $j=j^{\prime}$ or there exists $m \in M_{L}$ such that $j \uparrow m$ and $j^{\prime} \not \leq m$. We have the following results:

Proposition 9 Let $\emptyset \subset U, V \subseteq S, x \in U, y \in V$, and $|S|=s$.

1- For $c_{U, x} \neq c_{V, y}, c_{U, x} \delta c_{V, y}$ if and only if $\{x\}=\{y\} \subset V \subseteq U$.

2- The dependence relation $\delta$ (Definition 7) on the lattice $\mathcal{C}$ of all choice functions verifying

$(C)$ is isomorphic to the union of $s$ top truncated Boolean lattices $\left(2^{s-1}-1\right)$ and of an antichain of size $s$.

\section{Proof}

Let $\emptyset \subset U, V \subseteq S, x \in U, y \in V$.

1- Since $\mathcal{C}$ is atomistic $c_{U, x} \delta c_{V, y}$ (with $c_{U, x} \neq c_{V, y}$ ) if and only if there exists $c \in M_{\mathcal{C}}$ such that $c_{U, x} \uparrow c$ and $c_{V, y} \not \leq c$, so if $c_{U, x} \uparrow c$ and $y \notin c(V)$. By Proposition $8, c_{U, x} \uparrow c$ if and only if $U=\{x\}$ and $c=c^{x, x}$, or there exists $t \in U-x$ such that $c=c^{[x t, U], x}$. In the case $c=c^{x, x}$, we have $c_{V, y} \not \leq c^{x, x}$ if and only if $y \notin c^{x, x}(V)$ if and only if $\{x\}=\{y\}=V$. Then $c_{U, x}=c_{V, y}\left(=c_{x, x}\right)$ a contradiction with our assumption. Then $c=c^{[x t, U], x}$ and $y \notin c(V)$, 
that is equivalent to $\{x, t\} \subseteq V \subseteq U$ and $y=x$, and equivalent to $\{x\}=\{y\} \subset V \subseteq U$.

2 - It is clear that the relation $\delta$ is reflexive and antisymmetric. We show that it is a transitive relation. Let $\emptyset \subset U, V, W$ and $x \in U \cap V \cap W$ such that $c_{U, x} \delta c_{V, x}$ and $c_{V, x} \delta c_{W, x}$. By1-, one has $\{x\} \subset W \subseteq V \subseteq U$. Then $c_{U, x} \delta c_{W, x}$. So $\delta$ is an order relation.

For $x \in S$ and $U, V \in[x, S]$, we have $c_{U, x} \delta c_{V, x}$ if and only if $\{x\} \subset V \subseteq U$. Hence for every $x \in S$, the poset $\left\{c_{U, x},\{x\} \subset U \subseteq S,|U|>1\right\}$ is isomorphic to the top truncated Boolean lattice $\left(\underline{2}^{s-1}-1\right)$. If $x \neq y$, for all $U \in[x, S]$ and $V \in[y, S], c_{U, x}$ and $c_{V, y}$ are incomparable according to the order $\delta$, and then $\delta$ is isomorphic to the union of $s$ top truncated Boolean lattices $\left(\underline{2}^{s-1}-1\right)$ and of the antichain formed by the $s$ atoms $c_{x, x}, x \in S$.

We deduce the following theorem:

Theorem 1 The lattice $\mathcal{C}$ of all choice functions satisfying $(C)$ is atomistic and lower bounded (Definition 9.8).

\section{Proof}

We have already observed in Proposition 7 that the lattice $\mathcal{C}$ is atomistic (Definition 9.2). Since its dependence relation $\delta$ is an order it has no cycle. Then $\mathcal{C}$ is lower bounded (Definition 9.8).

We have the following corollary (recall that $|S|=s$ ):

Corollary 3 1- The lattice $\mathcal{C}$ is lower locally distributive, lower semi-modular and ranked (Definition 9).

2- The length of $\mathcal{C}$ is $s 2^{s-1}$ and the rank of $c \in \mathcal{C}$ is $r(c)=\sum_{A \subseteq S}|c(A)| . c \prec c^{\prime}$ in the lattice $\mathcal{C}$ if and only if $c \prec c^{\prime}$ in $\mathcal{S}$.

\section{Proof}

1- This assertion results from the implications between classes of lattices recalled in Section 2 (Lemma 2).

2- It is known that the length of a lower locally distributive lattice is the number of its join-irreducible elements. Then $l(\mathcal{C})$ equals the number of atoms of $\mathcal{C}$, i.e. $s 2^{s-1}$. Since $\mathcal{C} \subset \mathcal{S}$ is a ranked lattice having the same length that $\mathcal{S}$, its maximal chains are maximal chains of $\mathcal{S}$. Then $r(c)$ in $\mathcal{C}$ equals $r(c)$ in $\mathcal{S}$, i.e. $\sum_{A \subseteq S}|c(A)|$. Let $c, c^{\prime} \in \mathcal{C}$. $c \prec c^{\prime}$ in $\mathcal{C}$ if and only if $c^{\prime}=c \vee c_{U, x}$ i.e. if and only if $c \prec c^{\prime}$ in $\mathcal{S}$.

\subsection{The lattice $\mathcal{O}$ of choice functions verifying $(\mathrm{O})$}

A choice function $c$ on $S$ (of cardinality $s$ ) verifies the outcast axiom ( $\mathbf{O}$ ) (called also Nash's axiom) if:

$$
\text { for all } A, B \subseteq S, c(A) \subseteq B \subseteq A \text { implies } c(B)=c(A) \text {. }
$$

We denote by $\mathcal{O}$ or $\mathcal{O}(s)$ the set of all such choice functions.

A choice function satisfying $(\mathrm{O})$ is obviously idempotent. Then, the set $c\left(2^{S}\right)$ of the images of $c$ is the set of the "fixed points" of $c$, i.e., the family of the subsets $A$ of $S$ such that $c(A)=A$. We denote by $\mathcal{I}_{c}$ the set of the images of $c$. For $U \in \mathcal{I}_{c}$, we set $\mathcal{B}_{U}=\{X \subseteq S$ : $c(X)=U\}=c^{-1}(U)$. The set $\mathcal{B}_{c}=\left\{\mathcal{B}_{U}: U \in \mathcal{I}_{c}\right\}$ is the canonical partition of $2^{S}$ associated 
to $c$. Note that this set contains always $\mathcal{B}_{\emptyset}$ the set of subsets of $X$ such that $c(X)=\emptyset$.

Another characterization of the choice functions verifying $(\mathrm{O})$ will be useful. It needs the following definition:

Definition $19 A$ bouquet on $S$ is a family $\mathcal{B}$ of subsets of $S$ which is stable by intersection ( $X, Y \in \mathcal{B}$ implies $X \cap Y \in \mathcal{B})$ and convex $(X \subseteq Y \subseteq Z$, and $X, Z \in \mathcal{B}$ imply $Y \in \mathcal{B})$.

The root of a bouquet $\mathcal{B}$ is the set $U=\bigcap\{X, X \in \mathcal{B}\}$ of $\mathcal{B}$. A bouquet with root $U$ is denoted by $\mathcal{B}_{U}$.

It is clear that every interval $[U, V]=\{X \subseteq S: U \subseteq X \subseteq V\}$ is a bouquet, and we have the following obvious proposition:

Proposition 10 A family $\mathcal{B}$ of subsets of $S$ is a bouquet if and only if $\mathcal{B}$ is the union of intervals $\left[U, X_{i}\right], i=1, \ldots, p$.

Choice functions verifying $(\mathrm{O})$ are characterized by means of the notion of bouquet:

Proposition 11 Let $c$ be a choice function on $S, \mathcal{I}_{c}=\left\{U_{1}, \ldots, U_{q}\right\}$ the set of the images of $c$ and $\mathcal{B}_{c}=\left\{\mathcal{B}_{1}, \ldots, \mathcal{B}_{q}\right\}$ the partition associated with $c: X \in \mathcal{B}_{i}$ if $c(X)=U_{i}$. c satisfies axiom $(O)$ if and only if for all $i=1, \ldots, q, \mathcal{B}_{i}$ is a bouquet with root $U_{i}$.

\section{Proof}

Indeed, when $c$ satisfies $(\mathrm{O}), \mathcal{B}_{i}$ is the bouquet $\mathcal{B}_{U_{i}}=\bigcup_{k}\left[U_{i}, X_{k}\right]$, where for every $k, X_{k} \in \mathcal{B}_{i}$ and is maximal (i.e. $c\left(X_{k}\right)=U_{i}$ and $X_{k} \subseteq X$ implies $c(X) \neq U_{i}$ ). The converse property is obvious, since when $c(X)=U$ and $\mathcal{B}_{U}$ is a bouquet, $U \subseteq Y \subseteq X$ implies that $Y \in \mathcal{B}_{U}$, hence $c(Y)=U$.

So we obtain the following corollary:

Corollary 4 Let $c$ be a choice function on $S$ and $X \subseteq S$. If c verifies $(O)$ and $c(X)=\emptyset$, then for every $Y \subseteq X$ we have $c(Y)=\emptyset$ (i.e. $c^{-1}(\emptyset)$ is a bouquet). In particular if $c(S)=\emptyset$ then $c=0_{\mathcal{S}}$.

It is easy to prove that the union of two choice functions of $\mathcal{O}$ satisfies $(\mathrm{O})$ and it is obvious that the trivial choice functions $0_{\mathcal{S}}$ and $1_{\mathcal{S}}$ verify $(\mathrm{O})$. On the other hand the intersection of two choice functions of $\mathcal{O}$ does not necessarily verify (O). For instance with $S=\{1,2\}$, $c=\underline{1}, \underline{2}, \underline{12}$ and $c^{\prime}=1, \underline{2}, 1 \underline{2}$ satisfy $(\mathrm{O})$ but not $c \cap c^{\prime}=1, \underline{2}, 12$. But since $\mathcal{O}$ is an $\bigcup$ semilattice containing a least element, Lemma 1 gives the following result (where $\leq$ is the point-wise order between choice functions).

Proposition 12 The poset $(\mathcal{O}, \leq)$ of all choice functions on $S$ verifying $(O)$ is a lattice with $0_{\mathcal{S}}$ (respectively $1_{\mathcal{S}}$ ) as least (respectively greatest) element. It is a sub-U-semilattice (Definition 3.1) of the lattice $(\mathcal{S}, \leq)$ of all choice functions on $S$.

Henceforth, the lattice $(\mathcal{O}, \leq)$ will be simply denoted by $\mathcal{O}$ or $\mathcal{O}(s)$. FIG.4 represents the diagram of the lattice $\mathcal{O}(2)$ (the choice functions are written with the conventions described 
for FIG.3). One can note that $\mathcal{O}(2)$ is a ranked lattice but that it is neither lower nor upper semi-modular.

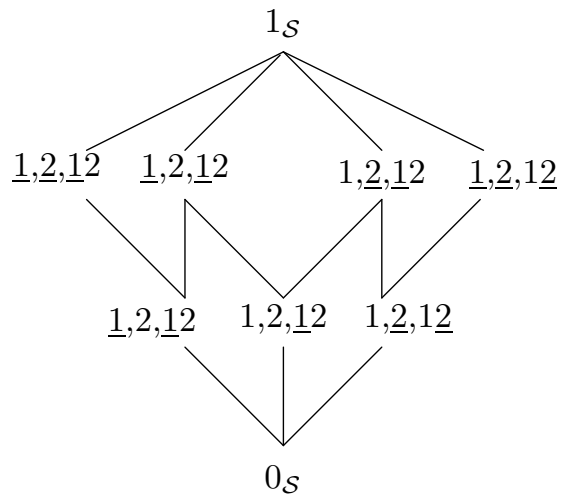

FIG.4 : The lattice $\mathcal{O}(2)$ of choice functions satisfying $(\mathrm{O})$.

It is easy to characterize the coatoms of the lattice $\mathcal{O}$.

Proposition 13 A choice function $c$ is a coatom (Definition 5.4) of the lattice $\mathcal{O}$ if and only if $c$ is a coatom $c^{U, x}$ of the lattice $\mathcal{S}$. Moreover the lattice $\mathcal{O}$ is coatomistic (Definition $9.3)$.

\section{Proof}

Let $c=c^{U, x}$ be a coatom of $S$, i.e. a choice function such that $c(U)=U-x$ and $c(X)=X$ for $X \neq U . c$ belongs to $\mathcal{O}$ since the images of $c$ are all the subsets $X \neq U$ for which $\mathcal{B}_{X}=\{X\}$, and the subset $U-x$ for which $\mathcal{B}_{U-x}=[U-x, U]$. Then $c$ is a coatom of $\mathcal{O}$, and since every choice function satisfying $(\mathrm{O})$ is contained in (at least) a coatom $c^{U, x}$ the converse is true. The lattice $\mathcal{O}$ being contained in the coatomistic lattice $\mathcal{S}$ and having the same coatoms is coatomistic too.

Since $\mathcal{O}$ is coatomistic, we have determined the meet-irreducible elements of the lattice $\mathcal{O}$. Now we search to determine the join-irreducible elements of this lattice, a not easy task except in the case of its atoms. In order to determine these atoms we introduce the following particular choice functions:

Definition 20 Let $\emptyset \subset U \subseteq S$. We define the choice function $c_{U}$ on $S$ by: $c_{U}(X)=U$ if $U \subseteq X \subseteq S$ and $c_{U}(X)=\emptyset$ if not.

Proposition $14 A$ choice function $c$ is an atom (Definition 5) of the lattice $\mathcal{O}$ if and only if there exists a non-empty subset $U$ of $S$ such that $c=c_{U}$.

\section{Proof}

First we prove that $c_{U} \in \mathcal{O}$ for every $\emptyset \subset U \subseteq S$. Indeed, the images of $c_{U}$ are $\emptyset$ and $U$. Since $\mathcal{B}_{\emptyset}=\bigcup_{x \in U}[\emptyset, S-x]$ and $\mathcal{B}_{U}=[U, S]$ are two bouquets, $c \in \mathcal{O}$ (by Proposition 11). In order to prove that $c=c_{U}$ is an atom we have to show that the only choice function in $\mathcal{O}$ less than $c$ is $0_{\mathcal{S}}$. Let $c^{\prime} \in \mathcal{O}$ such that $c^{\prime}<c$. For every $X \nsupseteq U, c^{\prime}(X)=\emptyset$ and for every $X \supseteq U, c^{\prime}(X) \subseteq U$. If $c^{\prime}(S)=c(S)=U$ then there exists $X$ with $U \subseteq X \subset S$ such that 
$c^{\prime}(X) \subset U$. This is impossible since $c^{\prime} \in \mathcal{O}$ and $c^{\prime}(S)=U \subseteq X \subset S$. Hence $c^{\prime}(S) \subset U$, and since $U \nsubseteq c^{\prime}(S), c^{\prime}\left(c^{\prime}(S)\right)=\emptyset$. Since $c^{\prime} \in \mathcal{O}, c^{\prime}$ is idempotent and then $c^{\prime}(S)=c^{\prime}\left(c^{\prime}(S)\right)=\emptyset$. So $c^{\prime}=0_{\mathcal{S}}$ (by Corollary 4) and we have proved that $c=c_{U}$ is an atom of $\mathcal{O}$.

Conversely let $c$ be an atom of $\mathcal{O}$. Then $c(S) \neq \emptyset$. We set $U=c(S)$. For every $X \supseteq U$, $c(X)=U$. Then $c_{U} \leq c$. But $c_{U}$ and $c$ are atoms so $c=c_{U}$.

Now we are going to determine another class of join-irreducible elements (Definition 5) of $\mathcal{O}$. We need the following definitions and properties.

Definition 21 A family $\left\{U_{1}, \ldots, U_{p}\right\}$ of subsets of $S$ is a Sperner family on $S$ if $U_{i}$ and $U_{j}$ are incomparable for all $i \neq j, i, j \in\{1, \ldots, p\}$.

An ordered Sperner family on $S$ is a Sperner family $\left\{U_{1}, \ldots, U_{p}\right\}$ ordered by a strict total order $>$. We denote it by $\left(U_{1}>U_{2}>\ldots>U_{p}\right)$.

The function $c_{U_{1}>U_{2}>\ldots>U_{p}}$ associated to the ordered Sperner family $\left(U_{1}>U_{2}>\ldots>U_{p}\right)$ is the function on $2^{S}$ defined by: $c_{U_{1}}>\ldots>U_{p}(X)=U_{i}$ if $X \in \mathcal{B}_{U_{i}}=\{X \subseteq S: \forall k=1, . ., i-1$, $U_{k} \nsubseteq \subseteq X$ and $\left.U_{i} \subseteq X\right\}$ and $c_{U_{1}}>\ldots>U_{p}(X)=\emptyset$ if $X \in \mathcal{B}_{\emptyset}=\left\{X \subseteq S: \forall k=1, \ldots, p, U_{k} \nsubseteq \subseteq X\right.$.

In particular, when the ordered Sperner family reduces to the empty set, $c_{\emptyset}=0_{\mathcal{S}}$.

For instance $(1235>14>234>45)$ is an ordered Sperner family on $S=\{1,2,3,4,5\}$.

Proposition 15 The choice function $c=c_{U_{1}}>\ldots>U_{p}$ associated to an ordered Sperner family $\left(U_{1}>\ldots>U_{p}\right)$ is a choice function satisfying the outcast axiom (O).

\section{Proof}

To prove this result we will successively demonstrate the following points:

1) For all $i \in\{1, \ldots, p\}, \mathcal{B}_{U_{i}}$ is a bouquet with root $U_{i}$.

2) The family $\mathcal{B}_{\emptyset}$ is a bouquet with root the empty set.

3) $\mathcal{B}_{\emptyset} \cup\left\{\mathcal{B}_{U_{i}}, i=1, \ldots, p\right\}$ is a partition of $2^{S}$.

Let $\left(U_{1}>\ldots>U_{p}\right)$ be an ordered Sperner family on $S$. For $i \in\{2, \ldots, p\}$ we denote by $\mathcal{T}_{i}$ the set of the minimal transversals of the family $\left\{U_{1}-U_{i}, \ldots, U_{i-1}-U_{i}\right\}$ (note that by definition $U_{k}-U_{i} \neq \emptyset$ for every $k<i$ ).

1) By definition $\mathcal{B}_{U_{1}}=\left[U_{1}, S\right]$ and we claim that $\mathcal{B}_{U_{i}}=\bigcup_{T \in \mathcal{T}_{i}}\left[U_{i}, S-T\right]$ for every $i=2, \ldots, p$. Let $i>1$ and $X \in \mathcal{B}_{U_{i}}$. Then $U_{i} \subseteq X$ and $U_{k} \nsubseteq X$ for every $k=1, \ldots, i-1$. Hence there exists $x_{k} \in U_{k}-U_{i}$ such that $X \subseteq S-x_{k}$. Then $X \subseteq S-\left\{x_{1}, \ldots, x_{i-1}\right\}$ with $\left\{x_{1}, \ldots, x_{i-1}\right\}$ transversal of $\left\{U_{1}-U_{i}, \ldots, U_{i-1}-U_{i}\right\}$. Let $T \in \mathcal{T}_{i}$ be a minimal transversal of $\left\{U_{1}-U_{i}, \ldots, U_{i-1}-U_{i}\right\}$ such that $T \subseteq\left\{x_{1}, \ldots, x_{i-1}\right\}$. Then $X \subseteq S-T$. So $X \in$ $\bigcup_{T \in \mathcal{T}_{i}}\left[U_{i}, S-T\right]$.

Conversely if $X \in \bigcup_{T \in \mathcal{T}_{i}}\left[U_{i}, S-T\right]$, then there exists $T \in \mathcal{T}_{i}$ such that $U_{i} \subseteq X \subseteq S-T$. Moreover one has $U_{k} \nsubseteq X$ for every $k=1, \ldots, i-1$. If not $U_{k} \subseteq X \subseteq S-T$, and then $U_{k} \cap T=\left(U_{k}-U_{i}\right) \cap T=\emptyset$ a contradiction with $T \in \mathcal{T}_{i}$. So $X \in \mathcal{B}_{U_{i}}$.

2) If $X \in \mathcal{B}_{\emptyset}, \emptyset \subseteq Y \subseteq X$ obviously implies $Y \in \mathcal{B}_{\emptyset}$. Then $\mathcal{B}_{\emptyset}$ is the bouquet equal to the union of intervals $\left[\emptyset, X_{i}\right]$ where $X_{i}$ is maximal in $\mathcal{B}_{\emptyset}$.

3) Let $\mathcal{B}_{U_{i}}$ the bouquet with root $U_{i}$. For all $i \neq j, \mathcal{B}_{U_{i}} \cap \mathcal{B}_{U_{j}}=\emptyset$. Indeed, if for example $i<j, X \in \mathcal{B}_{U_{i}}$ implies that $U_{i} \subseteq X$, and $X \in \mathcal{B}_{U_{j}}$ implies that $U_{i} \not \subset X$. On the other hand for every $X \in \mathcal{B}_{\emptyset}$ one has $U_{k} \nsubseteq \subseteq X$ for every $k$, then $\mathcal{B}_{\emptyset} \cap \mathcal{B}_{U_{k}}=\emptyset$ for every $k$. And since it is clear that for every $X \in 2^{S}, X \in \mathcal{B}_{U_{i}}$ for an $i \in\{1, \ldots, p\}$ or $X \in \mathcal{B}_{\emptyset}$, one has the result. Then the canonical partition $\mathcal{B}_{c}=\left\{\mathcal{B}_{U_{1}}, \ldots, \mathcal{B}_{U_{p}}, \mathcal{B}_{\emptyset}\right\}$ of $2^{S}$ associated to $c$ is formed of bouquets and by Proposition 11, $c \in \mathcal{O}$.

Henceforth we will simply denote $\mathcal{B}_{c}=\left\{\mathcal{B}_{1}, \ldots, \mathcal{B}_{p}, \mathcal{B}_{\emptyset}\right\}$. We will also need to consider $\mathcal{B}_{c}^{*}=\left\{\mathcal{B}_{1}, \ldots, \mathcal{B}_{p}\right\}$. 
Example $7 \quad c_{1235}>14>234>45$ is the choice function associated to the ordered Sperner family $1235>14>234>45$. It is represented by its set $\mathcal{B}_{c}^{*}=\left\{\mathcal{B}_{1}=\mathcal{B}_{1235}, \mathcal{B}_{2}=\mathcal{B}_{14}, \mathcal{B}_{3}=\mathcal{B}_{234}\right.$ and $\left.\mathcal{B}_{4}=\mathcal{B}_{45}\right\}$ of bouquets on FIG.5.

We are going to show that some choice functions $c_{U_{1}}>\ldots>U_{p}$ are join-irreducible elements of (O). In fact in Theorem 2 below, we will characterize such choice functions. But we need first to give some definitions and to prove several technical results. In particular we need to introduce a graph defined on the set $\mathcal{B}_{c}^{*}=\left\{\mathcal{B}_{U_{1}}, \ldots, \mathcal{B}_{U_{p}}\right\}$ of bouquets of $c$, different from $\mathcal{B}_{\emptyset}$, and to consider saturated sets and sources of this graph.

Definition 22 Let $c=c_{U_{1}}>\ldots>U_{p}$ be a choice function associated to an ordered Sperner family $\left(U_{1}>\ldots>U_{p}\right)$.

1. We define a digraph $G_{c}=\left(\mathcal{B}_{c}^{*}, \Delta\right)$ by: $\mathcal{B}_{c}^{*}=\left\{\mathcal{B}_{1}, \ldots, \mathcal{B}_{j}, \ldots, \mathcal{B}_{p}\right\}$ is the set of the bouquets of $c$ which are different from $\mathcal{B}_{\emptyset}\left(\mathcal{B}_{j}=c^{-1}\left(U_{j}\right)=\mathcal{B}_{U_{j}}\right)$; the relation $\Delta$ is defined by $\mathcal{B}_{k} \Delta \mathcal{B}_{j}$ if $k>j$ and there exists $X \in \mathcal{B}_{j}$ with $X \supset U_{k}$.

2. A subset $\mathcal{B}$ of $\mathcal{B}_{c}^{*}$ is a saturated set of the digraph $G_{c}=\left(\mathcal{B}_{c}^{*}, \Delta\right)$ if $\mathcal{B}_{k} \in \mathcal{B}$ and $\mathcal{B}_{k} \Delta \mathcal{B}_{j}$ imply $\mathcal{B}_{j} \in \mathcal{B}$.

3. A source of the digraph $G_{c}=\left(\mathcal{B}_{c}^{*}, \Delta\right)$ is a bouquet $\mathcal{B}_{i} \in \mathcal{B}_{c}^{*}$ such that there does not exist $\mathcal{B}_{j} \in \mathcal{B}_{c}^{*}(i<j)$ with $\mathcal{B}_{j} \Delta \mathcal{B}_{i}$.

4. We set $I=\{1, \ldots, p\}$. Let $\emptyset \subseteq J \subseteq I$. We define the choice function $c^{J}=c_{U_{1}>\ldots>U_{p}}^{J}$ on $S$ by: $c_{U_{1}>\ldots>U_{p}}^{J}(X)=U_{j}$ if $j \in J$ and $X \in \mathcal{B}_{j}$, and $c_{U_{1}>\ldots>U_{p}}^{J}(X)=\emptyset$ if not.

In other words $c^{J}$ is identical to $c$ on all the bouquets $\mathcal{B}_{j}$ where $j \in J$ and the choice is empty if not. In particular, $c^{\emptyset}=0_{\mathcal{S}}$ and $c^{I}=c$.

Example 8 The digraph $G_{c}=\left(\mathcal{B}_{c}^{*}, \Delta\right)$ for the choice function $c=c_{1235>14>234>45}$ is represented on FIG.5. $\mathcal{B}_{4}$ is a source of $G_{c}$ and $\left\{\mathcal{B}_{3}, \mathcal{B}_{2}, \mathcal{B}_{1}\right\}$ is a saturated set of $G_{c}$.

Remark 5 Observe that the digraph $G_{c}$ is contained in the graph of the linear order $\mathcal{B}_{p}>$ $\ldots>\mathcal{B}_{2}>\mathcal{B}_{1}$. So $\mathcal{B}_{p}$ is a source of $G_{c}$ and satisfies $\mathcal{B}_{k} \Delta \mathcal{B}_{1}, \forall k=2, \ldots, p$ (since $S \in \mathcal{B}_{1}$ ).

Note also that one can give an equivalent definition of the relation $\Delta$ :

$\mathcal{B}_{k} \Delta \mathcal{B}_{j}$ if and only if $j=1$, or $k>j$ and $\forall i=1, \ldots, j-1, U_{i} \not \subseteq U_{j} \cup U_{k}$.

Lemma 5 Let $c=c_{U_{1}>\ldots>U_{p}}$ be a choice function in $\mathcal{O}$, and $c^{\prime}$ a choice function. The two following properties are equivalent:

1. $c^{\prime}<c$ and $c^{\prime} \in \mathcal{O}$,

2. There exists $J \subset I=\{1, \ldots, p\}$ such that $c^{\prime}=c_{U_{1}>\ldots>U_{p}}^{J}$ and $\mathcal{B}^{J}=\left\{\mathcal{B}_{j}, j \in J\right\}$ is a saturated set of the digraph $G_{c}$.

In particular $c^{\prime} \prec c$ and $c^{\prime} \in \mathcal{O}$ if and only if $c^{\prime}=c^{I-j}$ and $\mathcal{B}^{I-j}$ is a saturated set of the digraph $G_{c}$.

\section{Proof}

1. $\Longrightarrow 2$.

a)- Assume that $c^{\prime}<c$ and $c^{\prime}$ not identical to $c$ on a bouquet $\mathcal{B}_{j}$. We are going to show that for every $X \in \mathcal{B}_{j}, c^{\prime}(X)=\emptyset$. By definition of the choice function $c$ there exists $X \in \mathcal{B}_{j}$ such that $c^{\prime}(X) \subset U_{j} . c\left(c^{\prime}(X)\right)=U_{i}$ (with $i \neq j$ ) is impossible since it would imply $U_{i} \subseteq c^{\prime}(X) \subset U_{j}$, a contradiction with the hypothesis that $\left(U_{1}, \ldots, U_{p}\right)$ is a Sperner 
family. Then $c\left(c^{\prime}(X)\right)=\emptyset$ and $c^{\prime}(X)=c^{\prime}\left(c^{\prime}(X)\right) \subseteq c\left(c^{\prime}(X)\right)=\emptyset$ imply $c^{\prime}(X)=\emptyset$. Since $c^{\prime}(X)=\emptyset \subset U_{j} \subseteq X$ and $c^{\prime} \in \mathcal{O}$, one has as well $c^{\prime}\left(U_{j}\right)=\emptyset$. Let now $Y \in \mathcal{B}_{j}$ with $Y \neq X$. $c^{\prime}(Y)=U_{j}$ is impossible since then $c^{\prime}\left(U_{j}\right)=c^{\prime}\left(c^{\prime}(Y)\right)$ would be equal to $U_{j} \neq \emptyset$. Then $c^{\prime}(Y) \subset U_{j}$ and like above $c^{\prime}(Y)=\emptyset$.

b)- Assume that $\mathcal{B}^{J}$ is not saturated. There exists $\mathcal{B}_{j} \in \mathcal{B}^{J}, \mathcal{B}_{i} \in \mathcal{B}_{c}$ with $\mathcal{B}_{j} \Delta \mathcal{B}_{i}$ and $\mathcal{B}_{i} \notin \mathcal{B}^{J}$. Then one has $c^{J}(X)=\emptyset$ for every $X \in \mathcal{B}_{i}$. But $\mathcal{B}_{j} \Delta \mathcal{B}_{i}$ implies the existence of $X \in \mathcal{B}_{i}$ such that $X \supset U_{j}$. Hence $\emptyset=c^{J}(X) \subset U_{j} \subset X$, but since $c^{J}\left(U_{j}\right)=U_{j}$, one contradicts $c \in \mathcal{O}$.

2 . $\Longrightarrow 1$.

Let $c^{\prime}=c_{U_{1}>\ldots>U_{p}}^{J}$ be a choice function associated with $\mathcal{B}^{J}$ a saturated subset of the digraph $G_{c}$. It is obvious that $c^{\prime}<c$. We claim that $c^{\prime}(X) \subseteq Y \subseteq X$ implies $c^{\prime}(X)=c^{\prime}(Y)$, i.e. $c^{\prime} \in \mathcal{O}$.

If $X \in \mathcal{B}_{j} \in \mathcal{B}^{J}$ (or $X \in \mathcal{B}_{\emptyset}$ ), $c^{\prime}(X)=c(X)=U_{j}$ (or $c^{\prime}(X)=\emptyset$ ). $\mathcal{B}_{j}$ convex implies $Y \in \mathcal{B}_{j}$ and then $c^{\prime}(Y)=U_{j}$ (or $\emptyset$ ).

If $X \in \mathcal{B}_{i}$ and $\mathcal{B}_{i} \notin \mathcal{B}^{J}, c^{\prime}(X)=\emptyset$. One has two cases:

a) $-Y \in \mathcal{B}_{k}$ with $\mathcal{B}_{k} \notin \mathcal{B}^{J}$. Then $c^{\prime}(Y)=\emptyset$ by the definition of $c^{\prime}=c_{U_{1}>\ldots>U_{p}}^{J}$.

b)- $Y \in \mathcal{B}_{j}$ with $\mathcal{B}_{j} \in \mathcal{B}^{J}$. Then $j<i$ is impossible since $U_{j} \subseteq Y \subset X$ would imply a contradiction with $X \in \mathcal{B}_{i}$. If $j>i$, then there exists $X \in \mathcal{B}_{i}$ with $X \supset U_{j}$, i.e. $\mathcal{B}_{j} \Delta \mathcal{B}_{i}$. But since $\mathcal{B}^{J}$ is saturated one has $\mathcal{B}_{i} \in \mathcal{B}^{J}$, a contradiction. So this second case is impossible.

Now we can give a characterization of some join-irreducible elements of the lattice $\mathcal{O}$. By remark $5, \mathcal{B}_{p}$ is always a source of the digraph $G_{c}$. The following result shows that the $c_{U_{1}>\ldots>U_{p}}$ join-irreducible elements of $\mathcal{O}$ are characterized by the fact that $\mathcal{B}_{p}$ is the unique source of $G_{c}$.

Theorem 2 Let $\left(U_{1}>\ldots .>U_{p}\right)$ be an ordered Sperner family on $S$ and $G_{c}=$ $\left(\left\{\mathcal{B}_{1}, \ldots, \mathcal{B}_{p}\right\}, \Delta\right)$ the digraph associated to the choice function $c=c_{U_{1}>\ldots>U_{p}}$. The two following properties are equivalent:

1. $c=c_{U_{1}>\ldots>U_{p}} \in J_{\mathcal{O}}$,

2. the digraph $G_{c}$ has $\mathcal{B}_{p}$ as unique source.

Moreover in this case the choice functions $0_{\mathcal{S}}, c_{U_{1}}, c_{U_{1}>U_{2}}, \ldots, c_{U_{1}}>\ldots>U_{p}$ form a covering chain of join-irreducible elements (Definition 5) of the lattice $\mathcal{O}: 0_{\mathcal{S}} \prec c_{U_{1}} \prec c_{U_{1}>U_{2}} \prec \ldots \prec$ $c_{U_{1}}>\ldots>U_{p}$.

In particular, $c=c_{U_{1}>\ldots>U_{p}} \in J_{\mathcal{O}}$ if the sets $U_{1}, \ldots, U_{p}$ are pairwise disjoint, and for $p=2$, $c_{U_{1}>U_{2}}$ is always a join-irreducible element covering $c_{U_{1}}$.

\section{Proof}

By Lemma $5, c^{\prime} \prec c$ in $\mathcal{O}$ if and only if $c^{\prime}=c^{I-j}(I=\{1, \ldots, p\})$ with $\mathcal{B}^{I-j}$ saturated set of the digraph $G_{c}$. Now it is obvious that $\mathcal{B}^{I-j}$ is saturated if and only if $\mathcal{B}_{j}$ is a source of $G_{c}$. Since $\mathcal{B}_{p}$ is a source of $G_{c}, \mathcal{B}^{I-p}$ is saturated and $c^{\prime}=c_{U_{1}>\ldots>U_{p-1}}$ is covered by $c$. Then $c$ is join-irreducible if and only if $c^{\prime}$ is the unique choice function covered by $c$ and if and only if $\mathcal{B}_{p}$ is the unique source of $G_{c}$.

Now take $c^{\prime}=c_{U_{1}>\ldots>U_{p-1}}$. Since the digraph $G_{C^{\prime}}$ is the restriction of the digraph $G_{c}$ to $\left\{\mathcal{B}_{1}, \ldots, \mathcal{B}_{p-1}\right\}$, it has also a unique source namely $\mathcal{B}_{p-1}$. Then iterating the above reasoning one obtains that $c_{U_{1}>\ldots>U_{p-2}}$ is join-irreducible and finally the stated result. If $U_{i} \cap U_{j}=\emptyset$ for all $i \neq j$, it is easy to check that $\mathcal{B}_{p}$ is the unique source of $G_{c}$. If $p=2$, it is obvious that $\mathcal{B}_{2}$ is the unique source of $G_{c}$. 
Example 9 The digraph $\mathcal{B}_{c}$ associated to the choice function $c=c_{1235}>14>234>45$ has a unique source (FIG.5). By Theorem 2, there exists a covering chain of join-irreducible elements of the lattice $\mathcal{O}$ represented on this same Figure.

Remark 6 The set $J_{\mathcal{O}}$ of join-irreducible elements of the lattice $\mathcal{O}$ contains strictly the set of join-irreducible $c_{U_{1}}>\ldots>U_{p}$ characterized in Theorem 2. Indeed let us consider the choice function defined on $S=\{1,2,3\}$ by $c(123)=123, c(12)=c(1)=1, c(23)=c(2)=2$ and $c(13)=c(3)=3$. It is easy to see that $c$ is join-irreducible in $\mathcal{O}$, but $c$ cannot be obtained as $c_{U_{1}>\ldots>U_{p}}$. Moreover in the lattice $\mathcal{O}(3)$ one can find a maximal chain of length (Definition 8) 5 from $0_{\mathcal{S}}$ to $1_{\mathcal{S}}$ passing by $c$ and another maximal chain from $0_{\mathcal{S}}$ to $1_{\mathcal{S}}$ of length 7 . Then for $s \geq 3, \mathcal{O}(s)$ is not a ranked lattice (Definition 9). One can show that the maximum length of a maximal chain in $\mathcal{O}(s)$ is $2^{s}-1$.

The following theorem summarizes the properties of the lattice $\mathcal{O}$ (recall that $|S|=s$ ):

Theorem 3 The lattice $\mathcal{O}$ of all choice functions satisfying axiom $(O)$ is coatomistic (Definition 9.3) (with the coatoms $c^{U, x}$ of the lattice $\mathcal{S}$ as coatoms). Its join-irreducible elements contain all the choice functions $c=c_{U_{1}}>\ldots>U_{p}$ such that the associated digraph $G_{c}=\left(\left\{\mathcal{B}_{1}, \ldots, \mathcal{B}_{p}\right\}, \Delta\right)$ has $\mathcal{B}_{p}$ as unique source. The length of $\mathcal{O}$ is $2^{s}-1$. For $s=2$ the lattice $\mathcal{O}(2)$ is ranked (Definition 9.7) but neither upper semi-modular nor lower semi-modular (Definition 9.5). For $s \geq 3$ the lattice $\mathcal{O}(s)$ is not ranked. 


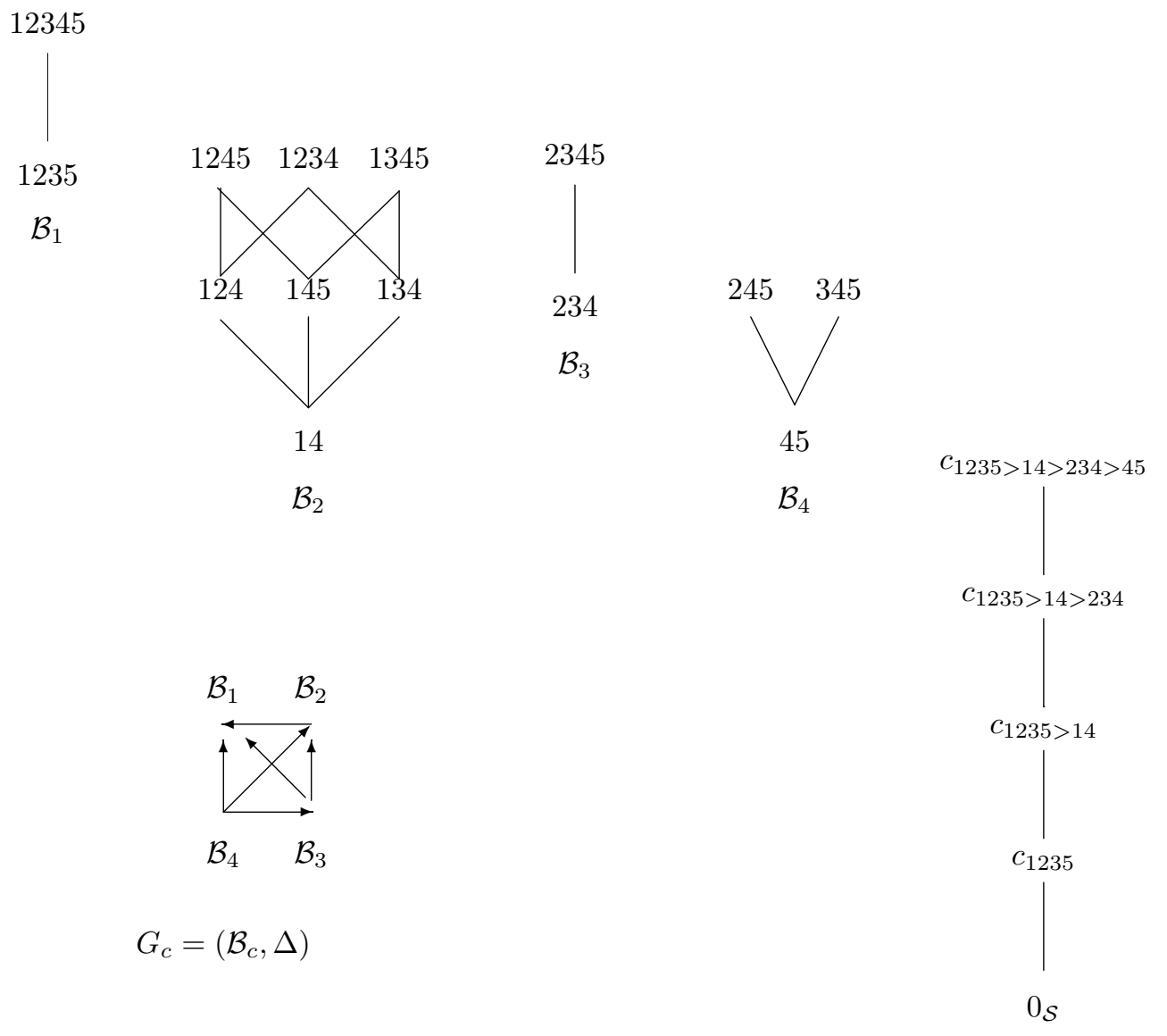

FIG.5: The choice function associated to $(1235>14>234>45)$, and the associated covering chain of join-irreducible elements of $\mathcal{O}$.

\section{The consensus of choice functions}

As said in the introduction we are going now to use the previous results on lattices of choice functions and the general results of the latticial consensus theory to derive results on the consensus of choice functions. We will consider successively the axiomatic approach (Section 5.1) then the metric approach (Section 5.2). In each case we will begin by giving some results of the latticial theory then we will apply them to the consensus of choice functions. We shall use the following definitions and notations:

Let $L$ be a finite lattice.

A $n$-profile or simply profile of $L$ is a $n$-tuple $\Pi=\left(x_{1}, \ldots, x_{i}, \ldots, x_{n}\right)$ of elements of $L$. We denote by $L^{n}$ the set of all $n$-profiles of $L$.

The consensus problem on $L$ consists of summarizing arbitrary profiles $\Pi$ of $L$ by one or several elements of $L$.

A consensus function $F$ on $L$ is a map from $L^{n}$ into $L$ or $2^{L}$, that is for every $\Pi$ in $L^{n}$, $F(\Pi)$ belongs to $L$ or is a subset of $L$. The problem is to find "good" consensus functions. 


\subsection{The axiomatic approach}

\subsubsection{Axiomatic latticial consensus theory}

First let us define some classes of latticial consensus functions $F: L^{n} \mapsto L$ ( $L$ lattice). A federation (called also simple game) on $N=\{1, \ldots, i, \ldots, n\}$ is a family $\mathcal{F}$ of subsets of $N$ satisfying $[G \in \mathcal{F}, K \supseteq G]$ imply $[K \in \mathcal{F}]$.

Definition 23 1. A federation consensus function is a consensus function $F$ on $L$ such that there exists a federation $\mathcal{F}$ for which $F(\Pi)=\bigvee_{K \in \mathcal{F}}\left(\bigwedge_{i \in K} x_{i}\right)$ for every $\Pi$ in $L^{n}$.

2. A meet-projection consensus function is a consensus function for which there exists $K \subseteq N(K \neq \emptyset)$ such that for each $\Pi$ in $L^{n}, F(\Pi)=\bigwedge_{i \in K} x_{i}$.

In particular, $F(\Pi)=x_{i}$ is a projection when $|K|=1$, and $F(\Pi)=\bigwedge_{i \in N} x_{i}$ is the Pareto (or unanimity) consensus function when $K=N$.

Note that the meet-projection consensus functions are federation consensus functions (take $\mathcal{F}=\{G \subseteq N: K \subseteq G\}$ ). In social choice theory the meet-projection (respectively projection) functions are often called oligarchic (respectively dictatorial ${ }^{7}$ ).

Using the dual operations on the lattice $L$ one defines dual consensus functions:

Definition 24 1. A co-federation consensus function is a consensus function $F$ on $L$ such that there exists a federation $\mathcal{F}$ for which $F(\Pi)=\bigwedge_{K \in \mathcal{F}}\left(\bigvee_{i \in K} x_{i}\right)$ for every $\Pi$ in $L^{n}$.

2. A join-projection consensus function is a consensus function for which there exists $K \subseteq N(K \neq \emptyset)$ such that for each $\Pi$ in $L^{n}, F(\Pi)=\bigvee_{i \in K} x_{i}$.

In particular $F(\Pi)=x_{i}$ is a projection when $|K|=1$, and $F(\Pi)=\bigvee_{i \in N} x_{i}$ is the co-Pareto consensus function when $K=N$.

Note that join-projection consensus functions are co-federation consensus functions (take $\mathcal{F}=\{G \subseteq N: K \subseteq G\})$. These functions can be also called co-oligarchic.

Next we give some Arrovian axioms of the latticial consensus theory.

Let $j$ be a join-irreducible element of the lattice $L$. We denote by $N_{j}(\Pi)$ the set $N_{j}(\Pi)=$ $\left\{i \in N: j \leq x_{i}\right\}$.

Definition $25 A$ consensus function $F$ on the lattice $L$ is:

1. $J_{L}$-neutral monotonic if for all $j$, $j^{\prime}$ in $J_{L}$ and $\Pi$, $\Pi^{\prime}$ in $L^{n}$, one has: $\left[N_{j}(\Pi) \subseteq\right.$ $\left.N_{j^{\prime}}\left(\Pi^{\prime}\right)\right] \Longrightarrow\left[j \leq F(\Pi) \Longrightarrow j^{\prime} \leq F\left(\Pi^{\prime}\right)\right]$.

2. $J_{L}$-decisive if for every $j$ in $J_{L}$ and for all $\Pi$, $\Pi^{\prime}$ in $L^{n},\left[N_{j}(\Pi)=N_{j}\left(\Pi^{\prime}\right)\right] \Longrightarrow[j \leq$ $\left.F(\Pi) \Longleftrightarrow j \leq F\left(\Pi^{\prime}\right)\right]$.

\footnotetext{
${ }^{7}$ In fact in social choice theory one has to consider preference agregation functions that are absolute dictatorial (i.e. projections) and preference agregation functions that are dictatorial. But we will not have to make this distinction here.
} 
3. Paretian if for each $\Pi$ in $L^{n}, \bigwedge_{i \in N} x_{i} \leq F(\Pi)$.

We define below dual axioms bearing on the meet-irreducible elements on $L$. For $m$ in $M_{L}$, $N_{m}(\Pi)=\left\{i \in N: m \geq x_{i}\right\}$.

Definition 26 A consensus function $F$ on the lattice $L$ is:

1. $M_{L}$-neutral monotonic if for all $m, m^{\prime}$ in $M_{L}$ and $\Pi, \Pi^{\prime}$ in $L^{n}$, one has: $\left[N_{m}(\Pi) \subseteq\right.$ $\left.N_{m^{\prime}}\left(\Pi^{\prime}\right)\right] \Longrightarrow\left[m \geq F(\Pi) \Longrightarrow m^{\prime} \geq F\left(\Pi^{\prime}\right)\right]$.

2. $M_{L}$-decisive if for every $m$ in $M_{L}$ and for all $\Pi$, $\Pi^{\prime}$ in $L^{n},\left[N_{m}(\Pi)=N_{m}\left(\Pi^{\prime}\right)\right] \Longrightarrow$ $\left[m \geq F(\Pi) \Longleftrightarrow m \geq F\left(\Pi^{\prime}\right)\right]$.

3. co-Paretian if for each $\Pi$ in $L^{n}, \bigvee_{i \in N} x_{i} \geq F(\Pi)$.

The consensus functions defined on a lattice $L$ depend on the chosen axioms and on the structural properties of the considered lattice, and in particular on the properties of the dependence relations $\delta$ (defined on the set $J_{L}$ of all join-irreducible elements of $L$ ) and $\beta$ (defined on the set $M_{L}$ of its meet-irreducible elements). The following theorem give some general results of the axiomatic latticial consensus theory (see [15], [21]).

Theorem 4 Let $L$ be a lattice and $F$ be a consensus function on $L$.

1. If $L$ is distributive (Definition 9.1), then $F$ is $J_{L}$-neutral monotonic if and only if $F$ is a federation consensus function.

2. If $L$ is not distributive, then $F$ is $J_{L}$-neutral monotonic and Paretian if and only if $F$ is a meet-projection.

3. If the dependence relation $\delta$ on $J_{L}$ (Definition 7.1) is strongly connected, then $F$ is $J_{L}$-decisive and Paretian if and only if $F$ is a meet-projection consensus function.

Note that the condition on the dependence relation $\delta$ in point 3 of the above Theorem implies that $L$ is not distributive (indeed, a lattice $L$ is distributive if and only if the dependence relation $\delta$ on $J_{L}$ is the order relation on $J_{L}$ (cf. [23]).

A dual theorem exists using dual axioms and the dual dependence relation $\beta$ defined on the set $M_{L}$ of all meet-irreducible elements of $L$. In particular one obtains a characterization of the co-federation and join-projection consensus functions.

Theorem 5 Let $L$ be a lattice and $F$ be a consensus function on $L$.

1. If $L$ is distributive (Definition 9.1), then $F$ is $M_{L}$-neutral monotonic if and only if $F$ is a co-federation consensus function.

2. If $L$ is not distributive, then $F$ is $M_{L}$-neutral monotonic and co-Paretian if and only if $F$ is a join-projection.

3. If the dependence relation $\beta$ on $M_{L}$ (Definition 7.2) is strongly connected, then $F$ is $M_{L}$-decisive and co-Paretian if and only if $F$ is a join-projection consensus function.

Remark 7 If a lattice $L$ is distributive any co-federation consensus function is equal to a federation consensus function. So in this case Theorem 4 and 5 (points 1) give two characterizations of the same class of consensus functions.

We now deal with the consensus functions of choice functions verifying the axioms $(\mathrm{H}),(\mathrm{C})$ or $(\mathrm{O})$. Then $\Pi=\left(c_{1}, \ldots, c_{i}, \ldots, c_{n}\right)$ is a profile of choice functions all belonging either to $\mathcal{H}$, or to $\mathcal{C}$ or to $\mathcal{O}$. A consensus function is now -for instance in the case of $\mathcal{H}$ - a function $F$ from $\mathcal{H}^{n}$ to $\mathcal{H}$. Then $F(\Pi)$ is denoted by $c$ (and $F\left(\Pi^{\prime}\right)$ by $\left.c^{\prime}\right)$. 


\subsubsection{The consensus of choice functions satisfying the heredity property (H)}

Recall (Proposition 2) that the poset $(\mathcal{H}, \leq)$ of choice functions satisfying $(\mathrm{H})$ is a distributive lattice (Definition 9.1). Since (Proposition 3) $J_{\mathcal{H}}=\left\{c_{[x, A]}, x \in A \subseteq S\right\}$ and (Proposition 4) $M_{\mathcal{H}}=\left\{c^{[x, A]}, x \in A \subseteq S\right\}$, it is straightforward to check that the axioms given in Definition 25 and Definition 26 become the following ones:

A consensus function $F$ on $\mathcal{H}$ is:

- $J_{\mathcal{H}}$-neutral monotonic if for all $\Pi, \Pi^{\prime}$ in $\mathcal{H}^{n}$, for all subsets $A, B$ of $S$, for every $x$ in $A$ and for every $y$ in $B,\left[\left\{i \in N: \forall X\right.\right.$ with $\left.x \in X \subseteq A, x \in c_{i}(X)\right\} \subseteq\{i \in N: \forall Y$ with $\left.\left.y \in Y \subseteq B, y \in c_{i}^{\prime}(Y)\right\}\right]$ implies $[\forall X$ with $x \in X \subseteq A, x \in c(X)] \Longrightarrow[\forall Y$ with $\left.y \in Y \subseteq B, y \in c^{\prime}(Y)\right]$.

- $M_{\mathcal{H}}$-neutral monotonic if for all $\Pi, \Pi^{\prime}$ in $\mathcal{H}^{n}$ for all subsets $A, B$ of $S$, for every $x$ in $A$ and for every $y$ in $B,\left[\left\{i \in N: \forall X\right.\right.$ with $\left.A \subseteq X, x \notin c_{i}(X)\right\} \subseteq\{i \in N: \forall Y$ with $\left.\left.B \subseteq Y, y \notin c_{i}^{\prime}(Y)\right\}\right]$ implies $[\forall X$ with $A \subseteq X, x \notin c(X)] \Longrightarrow[\forall Y$ with $B \subseteq Y$, $\left.y \notin c^{\prime}(Y)\right]$.

- Paretian if for every $\Pi$ in $\mathcal{H}^{n}$, for every subset $A$ of $S$ and for every $x$ in $A,[\forall i \in N$, $\left.x \in c_{i}(A)\right] \Longrightarrow[x \in c(A)]$.

- co-Paretian if for every $\Pi$ in $\mathcal{H}^{n}$, for every subset $A$ of $S$ and for every $x$ in $A$, $\left[\forall i \in N, x \notin c_{i}(A)\right] \Longrightarrow[x \notin c(A)]$.

By Theorem 4.1, Theorem 5.1 and Remark 7 the following result holds:

Theorem 6 A consensus function $F$ on $\mathcal{H}$ is $J_{\mathcal{H}}$-neutral monotonic and Paretian if and only if $F$ is $M_{\mathcal{H}}$-neutral monotonic and co-Paretian and if and only if $F$ is a federation consensus function (i.e. if there exists a federation $\mathcal{F}$ on $N$ such that for every $\Pi$ in $\mathcal{H}^{n}$ and for every $\left.A \subseteq S, c(A)=\bigcup_{K \in \mathcal{F}}\left[\bigcap_{i \in K} c_{i}(A)\right]\right)$.

Remark 8 In the case of the Boolean lattice (Definition 9.4) $\mathcal{S}$ of all choice functions on $S$ one could write the same result but with axioms of neutrality monotonicity using the atoms and the coatoms of $\mathcal{S}$, i.e. the choice functions $c_{A, x}$ and $c^{A, x}$. For instance, in the case of $J_{\mathcal{S}}$, this axiom is the same that the axiom of $J_{\mathcal{C}}$-neutrality monotonicity given just below.

\subsubsection{Consensus of choice functions satisfying the concordance axiom (C)}

Recall (Theorem 1 ) that the poset $(\mathcal{C}, \leq)$ of choice functions satisfying $(\mathrm{C})$ on $S$ is a lower bounded lattice (Definition 9.8) (not distributive) and that its dependence relation $\delta$ is not strongly connected (Proposition 9). Since (Proposition 7) $J_{\mathcal{C}}=J_{\mathcal{S}}$ the axiom of $J$-neutrality monotonicity (Definition 25) becomes:

A consensus choice function $c$ on $\mathcal{C}$ is $J_{\mathcal{C}}$-neutral monotonic if for all $\Pi, \Pi^{\prime}$ in $\mathcal{C}^{n}$, for all subsets $A, B$ of $S$, for every $x$ in $A$ and for every $y$ in $B$ one has:

$$
\left[\left\{i \in N: x \in c_{i}(A)\right\} \subseteq\left\{i \in N: y \in c_{i}^{\prime}(B)\right\}\right] \Longrightarrow\left[x \in c(A) \Longrightarrow y \in c^{\prime}(B)\right]
$$

Then Theorem 4 induces the following result: 
Theorem $7 A$ consensus function $F$ on $\mathcal{C}$ is $J_{\mathcal{C}}$-neutral monotonic and Paretian if and only if $F$ is oligarchic (there exists $K \subseteq N$ such that for every $\Pi \in \mathcal{C}$ and for every $A \subseteq S$, $\left.c(A)=\bigcap_{i \in K} c_{i}(A)\right)$.

Taking the corresponding axioms for $M_{\mathcal{C}}$ one would obtain a dual result, i.e. a characterization of the co-oligarchic consensus functions on $\mathcal{C}$.

\subsubsection{Consensus of choice functions satisfying the outcast axiom (O)}

Recall (Proposition 13) that $M_{\mathcal{O}}=M_{\mathcal{S}}=\left\{c^{A, x}: x \in A \subseteq S\right\}$, with $c^{A, x}(X)=A-x$ if $X=A$ and $c^{A, x}(X)=X$ if not. Definition 26 induces the following axioms:

A consensus choice function $c$ on $\mathcal{O}$ is:

- $M_{\mathcal{O}}$-neutral monotonic if for all $\Pi, \Pi^{\prime}$ in $\mathcal{O}^{n}$, for all subsets $A, B$ of $S$, for every $x$ in $A$ and for every $y$ in $B,\left[\left\{i \in N: x \notin c_{i}(A)\right\} \subseteq\left\{i \in N: y \notin c_{i}^{\prime}(B)\right\}\right]$ implies $[x \notin c(A)] \Longrightarrow\left[y \notin c^{\prime}(B)\right]$.

- co-Paretian if for every $\Pi$ in $\mathcal{O}^{n}$, for every $A$ in $S$ and for every $x$ in $A,[\forall i \in N$, $\left.x \notin c_{i}(A)\right] \Longrightarrow[x \notin c(A)]$.

By Theorem 5 the following result holds:

Theorem 8 A consensus function $F$ on $\mathcal{O}$ is $M_{\mathcal{O}}$-neutral monotonic and co-Paretian if and only if $F$ is co-oligarchic (i.e. if there exists $K \subseteq N$ such that for every $\Pi \in \mathcal{O}^{n}$ and for every $\left.A \subseteq S, c(A)=\bigcup_{i \in K} c_{i}(A)\right)$.

We conjecture that the dependence relation $\beta$ on $M_{\mathcal{O}}$ is strongly connected. If this conjecture was true, one could replace in Theorem 8 the $M_{\mathcal{O}}$-neutrality monotonicity axiom by the simpler $M_{\mathcal{O}}$-decisivity axiom.

\subsection{The metric approach}

\subsubsection{Metric latticial consensus theory}

In this approach we take as consensus of a profile $\Pi=\left(x_{1}, \ldots, x_{n}\right)$ of elements of a lattice $L$ an element $x$ minimizing the "remoteness" of $x$ to the elements of $\Pi$. If the remoteness is computed as $D(x)=\sum_{i=1}^{n} d\left(x, x_{i}\right)$, where $d$ is a metric defined on $L$, such an element is called a $d$-median of $\Pi$. Here we take as metric $d$ on $L$ the minimum path length metric in the (unoriented) covering graph $G(L)$ of $L: x$ and $y$ are linked by an edge in $G(L)$ if and only if $x \prec y$ or $y \prec x . d(x, y)$ is the minimum number of edges of a path of $G(L)$ between $x$ and $y$. Then a $d$-median of $\Pi$ will be simply called a median of $\Pi$. Such a median always exists (since $L$ is finite) but it is not necessarily unique. We shall denote by $\operatorname{Med}_{L}(\Pi)$ or simply $\operatorname{Med}(\Pi)$ the set of medians of $\Pi$ and we call median rule the consensus rule associating to each profile its set of medians (for the median rule in general, see e.g. [5]).

These notions are illustrated on FIG.1. This figure was already used to show the diagram of a lattice $L$. If one considers now that a link between two elements represents an edge and not a (directed) arc, this same Figure represents the covering graph $G(L)$ of $L$. On this figure the distance $d(k, b)$ is 3 (obtained for three different paths). The profile $\Pi=(b, g, h, k)$ has two medians $e$ and $g$ with $D(e)=D(g)=5$.

Note that in arbitrary lattices neither the computation of the minimum path length metric 
nor a fortiori the computation of the medians are necessarily easy. But in the case of lower semi-modular lattices (Definition 9.5) the minimum path length metric is given by the following formula: $d(x, y)=2 r(x \vee y)-r(x)-r(y)$ where $r$ is the rank function (Definition 9.7) on $L^{8}$. In particular for distributive lattices this formula becomes $d(x, y)=r(x \vee y)-r(x \wedge y)$. For instance on FIG.1 which represents a distributive lattice, $d(k, b)=r(g)-r(0)=3-0=3$. Theorem 9 below shows that the medians are easy to compute in a distributive lattice. We use the following notations:

For all $\Pi \in L^{n}$ and $j \in J_{L}$ (respectively $m \in M_{L}$ ) we denote by $n_{j}(\Pi)$ (respectively $n_{m}(\Pi)$ ) the number of elements of $N_{j}(\Pi)$ such that $n_{j}(\Pi)=\left|N_{j}(\Pi)\right|$ (respectively $N_{m}(\Pi)$ such that $\left.n_{m}(\Pi)=\left|N_{m}(\Pi)\right|\right)$.

Definition 27 Let $\Pi \in L^{n}$. We define by two equivalent ways the following elements:

- $c_{\Pi}=\bigvee\left\{\bigwedge_{i \in K} x_{i}: K \subseteq N,|K|>n / 2\right\}=\bigvee\left\{j \in J_{L}: n_{j}(\Pi)>n / 2\right\}$.

- $c_{\Pi}^{*}=\bigvee\left\{\bigwedge_{i \in K} x_{i}: K \subseteq N,|K| \geq n / 2\right\}=\bigvee\left\{j \in J_{L}: n_{j}(\Pi) \geq n / 2\right\}$.

- $c_{\Pi}^{\prime}=\bigwedge\left\{\bigvee_{i \in K} x_{i}: K \subseteq N,|K|>n / 2\right\}=\bigwedge\left\{m \in M_{L}: n_{m}(\Pi)>n / 2\right\}$

$c_{\Pi}$ and $c_{\Pi}^{*}$ define consensus functions on $L^{n}$ called the strict majority rule and the majority rule respectively. Indeed they generalize the usual majority rules defined for profiles $\Pi=$ $\left(R_{1}, \ldots, R_{n}\right)$ of binary relations defined on a set $E$. For instance the strict majority rule is $R_{M A J}(\Pi)=\bigcup\left\{(x, y) \in E^{2}:\left|N_{\Pi}(x, y)\right|>n / 2\right\}=\bigcup\left\{\bigcap_{i \in K} R_{i}, K \subseteq N,|K|>n / 2\right\}$.

Note that one always has $c_{\Pi} \leq c_{\Pi}^{*} \leq c_{\Pi}^{\prime}$, and so one can consider the two nested intervals $\left[c_{\Pi}, c_{\Pi}^{*}\right]$ and $\left[c_{\Pi}, c_{\Pi}^{\prime}\right]$.

We have the following results $(\mathrm{cf}[11])$ :

Theorem 9 Let $L$ be a lattice.

1. $L$ is distributive (Definition 9.1) if and only if for every $\Pi \in L^{n}, \operatorname{Med}(\Pi)=\left[c_{\Pi}, c_{\Pi}^{\prime}\right]=$ $\left[c_{\Pi}, c_{\Pi}^{*}\right]$.

2. $L$ is lower semi-modular (Definition 9.5) if and only if for every $\Pi \in L^{n}$ and $x \in$ $\operatorname{Med}(\Pi), x \leq c_{\Pi}^{\prime}$.

So in a distributive lattice the medians of a profile are exactly the elements in the interval determined by the two majority rules. Note that if $n$ is odd these two elements coincide and so there is a unique median. We shall show in Remark 10 below how to compute the medians in a distributive lattice.

Remark 9 In a lower semi-modular lattice one can give a lower bound for the medians of a profile (see [16]).

In the case of a distributive lattice the median rule, which associates to each profile its set of medians can be characterized. We need some definitions:

First we denote by $L^{*}$ the set $\bigcup_{n>0} L^{n}$ of all profiles of $n$ elements of $L$, where $n$ is an integer greater than 0 . A consensus rule is a map $F: L^{*} \mapsto 2^{L}-\{\emptyset\}$ associating to each profile a non-empty subset of $L$. If $\Pi=\left(x_{1}, \ldots, x_{n}\right)$ and $\Pi^{\prime}=\left(y_{1}, \ldots, y_{p}\right)$ are two profiles, $\Pi \Pi^{\prime}$ denotes the concatened profile $\left(x_{1}, \ldots x_{n}, y_{1}, \ldots, y_{p}\right)$.

A consensus rule $F$ is:

\footnotetext{
${ }^{8}$ In Lemma 2 it has been recalled that a lower semi-modular lattice is ranked.
} 
- consistent if $F(\Pi) \cap F\left(\Pi^{\prime}\right) \neq \emptyset$ implies that $F\left(\Pi^{\prime}\right)=F(\Pi) \cap F\left(\Pi^{\prime}\right)$ (for all $\Pi$, $\left.\Pi^{\prime} \in L^{*}\right)$.

- unanimous if $F(x, \ldots, x)=x$, for every $x \in L$.

- quasi-Condorcet if for every $\Pi=\left(x_{1}, \ldots, x_{n}\right) \in L^{*}$, for every $j \in J_{L}$ such that $n_{j}(\Pi)=n / 2,\left[x \vee j \in F(\Pi)\right.$ if and only if $\left.x \vee j^{-} \in F(\Pi)\right]$ (where $j^{-}$is the unique element covered by $j$ ).

We have the following result ([6], [18]), ([18] correcting an error in the statement of the quasi-Condorcet condition contained in [6]).

Theorem 10 Let $L$ be a distributive lattice (Definition 9.1). A consensus rule $L^{*} \mapsto 2^{L}$ $\{\emptyset\}$ is the median rule if and only if it is consistent, unanimous and quasi-Condorcet.

Remark 10 In order to compute the medians of a profile $\Pi \in L^{n}$ when $L$ is a distributive lattice one can use the following procedure (see [11]). First one computes the least median $c_{\Pi}$ by the formula $c_{\Pi}=\bigvee\left\{j \in J_{L}: n_{j}(\Pi)>n / 2\right\}$. Then one computes the set $E(\Pi)=\left\{j \in J_{L}\right.$ : $\left.n_{j}(\Pi)=n / 2\right\}$ (an empty set if $n$ is odd). The medians of $\Pi$ are all the elements $c_{\Pi} \vee(\bigvee G)$ where $G$ is any subset of $E(\Pi)$.

Example 10 We illustrate the computation of medians described in the above Remark in the case of the distributive lattice $L$ of FIG.1. We take $\Pi=(b, g, h, k)$. One has

$n=4$

$J_{L}=\{a, b, f, k\}$

$n_{a}=|\{g, h, k\}|=3, n_{b}=|\{b, g\}|=3, n_{k}=|\{g, k\}|=2, n_{f}=|\{h\}|=1$

$c_{\Pi}=\bigvee\left\{j \in J_{L}: n_{j}(\Pi)>2\right\}=a \vee b=e$

$E(\Pi)=\left\{j \in J_{L}: n_{j}(\Pi)=2\right\}=\{k\}$.

Since $|E(\Pi)|=1, \operatorname{Med}(\Pi)=\left\{c_{\Pi}, c_{\Pi} \vee k\right\}=[e, g]$. And one cheks that $c_{\Pi}^{*}=\bigvee\left\{j \in J_{L}\right.$ : $\left.n_{j}(\Pi) \geq 2\right\}=a \vee b \vee k=g=c_{\Pi}^{\prime}=\bigwedge\left\{m \in M_{L}: n_{m}(\Pi)>2\right\}$.

We shall illustrate how to apply the results of this section in the case of $\mathcal{H}$ and $\mathcal{C}$.

\subsubsection{The median rule for the choice functions satisfying the heredity axiom (H)}

First we precise the minimum path lenght metric on $\mathcal{H}$. Since $\mathcal{H}$ is a covering sublattice of $\mathcal{S}$ (Corollary 1) the distance between two choice functions in $\mathcal{H}$ is the same that their distance in $\mathcal{S}$. Then since $\mathcal{S}$ is a Boolean lattice (Definition 9.4), $d\left(c, c^{\prime}\right)=r\left(c \cup c^{\prime}\right)-r\left(c \cap c^{\prime}\right)=$ $\sum_{A \subseteq S}\left[\left|c \cup c^{\prime}(A)\right|-\left|c \cap c^{\prime}(A)\right|\right]$.

We use the same notations that in 5.2.1 except that for $c_{[x, A]}$ in $J_{\mathcal{H}}$, we write $n_{[x, A]}(\Pi)$ (instead of $n_{c_{[x, A]}}$ ). So for $\Pi \in \mathcal{H}^{n}$ and $c_{[x, A]} \in J_{\mathcal{H}}$ :

- $n_{[x, A]}(\Pi)=\left|\left\{i \in N: c_{[x, A]} \leq c_{i}\right\}\right|=\mid\left\{i \in N: x \in X \subseteq A\right.$ implies $\left.x \in c_{i}(X)\right\} \mid$

- $c_{\Pi}=\bigcup\left\{\bigcap_{i \in K} c_{i}: K \subseteq N,|K|>n / 2\right\}=\bigcup\left\{c_{[x, A]} \in J_{\mathcal{H}}: n_{[x, A]}>n / 2\right\}$

- $c_{\Pi}^{*}=\bigcup\left\{\bigcap_{i \in K} c_{i}: K \subseteq N,|K| \geq n / 2\right\}=\bigcup\left\{c_{[x, A]} \in J_{\mathcal{H}}: n_{[x, A]} \geq n / 2\right\}$ 
The set of medians of a profile $\Pi \in \mathcal{H}^{n}$ is denoted by $\operatorname{Med}_{\mathcal{H}}(\Pi)$.

We denote by $\mathcal{H}^{*}$ the set $\bigcup_{n>0} \mathcal{H}^{n}$ and consider the following axioms for a consensus rule $F: \mathcal{H}^{*} \mapsto 2^{\mathcal{H}}-\{\emptyset\}:$

- $F$ is consistent if $F(\Pi) \cap F\left(\Pi^{\prime}\right) \neq \emptyset$ implies that $F\left(\Pi \Pi^{\prime}\right)=F(\Pi) \cap F\left(\Pi^{\prime}\right)$ (for all $\Pi$, $\left.\Pi^{\prime} \in \mathcal{H}^{*}\right)$.

- $F$ is unanimous if $F(c, \ldots, c)=c$, for every $c \in \mathcal{H}$.

- $F$ is quasi-Condorcet if for every $\Pi \in \mathcal{H}^{*}$, for every $c_{[x, A]} \in J_{\mathcal{H}}$ such that $n_{[x, A]}(\Pi)=$ $n / 2,\left[c \cup c_{[x, A]} \in F(\Pi)\right.$ if and only if $\left.c \cup c_{[x, A]}^{-} \in F(\Pi)\right]$.

Then applying Theorems 9 and 10, and noting by $\left[c_{\Pi}, c_{\Pi}^{*}\right]_{\mathcal{H}}$ the interval determined by $c_{\Pi}$ and $c_{\Pi}^{*}$ in the lattice $\mathcal{H}$, one gets:

Theorem 11 Let $\mathcal{H}$ be the lattice of all choice functions verifying the heredity axiom.

- For every $\Pi \in \mathcal{H}^{n}, \operatorname{Med}_{\mathcal{H}}(\Pi)=\left[c_{\Pi}, c_{\Pi}^{*}\right]_{\mathcal{H}}$.

- A consensus rule $\mathcal{H}^{*} \mapsto 2^{\mathcal{H}}-\{\emptyset\}$ is the median rule associating to each profile $\Pi$ the set of medians $\operatorname{Med}_{\mathcal{H}}(\Pi)$ if and only if it is consistent, unanimous and quasi-Condorcet.

Example 11 Consider the lattice $\mathcal{H}(2)$ represented in FIG. 3 and take $\Pi=(\underline{1}, 2, \underline{12} ; \underline{1}, \underline{2}, \underline{12}$; $1, \underline{2}, 12 ; \underline{1}, \underline{2}, 1 \underline{2})$. In order to compute the medians of this profile one can remark that the lattice $\mathcal{H}(2)$ is isomorphic to the lattice of FIG.1, and that in this isomorphism the above profile $\Pi$ corresponds to the profile $\Pi=(b, g, h, k)$ considered in Example 10. Since the medians of this last profile are $e$ and $g$ the medians of the first profile are $\underline{1}, \underline{2}, 12$ and $\underline{1}, \underline{2}, \underline{12}$. Note that in this example there exists no other choice functions between the two medians of $\Pi$. More generally, since $\mathcal{S}$ is a Boolean lattice, the interval $\left[c_{\Pi}, c_{\Pi}^{*}\right]$ is the set of medians of $\Pi$ in $\mathcal{S}$. When $\Pi \in \mathcal{H}^{n}$, the set $\left[c_{\Pi}, c_{\Pi}^{*}\right]_{\mathcal{H}}$ of medians of $\Pi$ in $\mathcal{H}$ is $\left[c_{\Pi}, c_{\Pi}^{*}\right] \cap \mathcal{H}$, which is generally a strict subset of $\left[c_{\Pi}, c_{\Pi}^{*}\right]$.

\subsubsection{The median rule for choice functions satisfying the concordance axiom (C)}

Recall (Corollary 3 ) that the lattice $\mathcal{C}$ of choice functions on $S$ satisfying the concordance axiom $(\mathrm{C})$ is lower semi-modular (Definition 9.5). We denote by $\operatorname{Med}_{\mathcal{C}}(\Pi)$ the set of medians of a profile $\Pi \in \mathcal{C}^{n} . c_{\Pi}^{\prime}=\bigcap\left\{c \in M_{\mathcal{C}}: n_{c}(\Pi)>n / 2\right\}$ where (Corollary 2) $M_{\mathcal{C}}=\left\{c^{x, x}, x \in\right.$ $S\} \cup\left\{c^{[x y, V], x}, x, y \in V \subseteq S\right\}$.

By applying Theorem 9 we have the following result:

Proposition 16 For every $\Pi \in \mathcal{C}^{n}$, and for every $c \in \operatorname{Med}_{\mathcal{C}}(\Pi), c \leq c_{\Pi}^{\prime}$.

According to Remark 9 it would be also possible to obtain a lower bound for the medians of $\Pi$ in $\mathcal{C}$. 


\section{Conclusion}

In this paper we have shown how to apply the general results of the latticial consensus theory to the consensus problems for classes of choice functions which are lattices, and in particular for the choice functions satisfying the heritage, concordance or outcast axioms. This approach need to study the structural properties of such lattices, what has been done in Sections 3 and 4.

Some results obtained by this approach have been given in Section 5 . One can compare these results with other results obtained by the more classical approach followed by the russian school and summarized in Aizerman and Aleskerov's book ([1]). In fact, this comparison can concern only the axiomatic approach since the metric approach is not considered (at least) in this book ${ }^{9}$. The main difference is that our axioms on consensus functions are defined with respect to the irreducible elements of the lattice of choice functions considered, whereas their axioms are always defined with respect to the choice functions $c_{A, x}$. These choice functions are not explicitly considered in their work, but all their consensus functions satisfy what they call the local property, namely:

For all $\Pi, \Pi^{\prime}$, for every $A \subseteq S$ and for every $x \in A\left[\left\{i \in N: x \in c_{i}(A)\right\}=\{i \in N\right.$ : $\left.\left.x \in c_{i}^{\prime}(A)\right\}\right] \Longrightarrow\left[x \in c(A) \Longleftrightarrow x \in c^{\prime}(A)\right]$.

Since $J_{\mathcal{S}}=J_{\mathcal{C}}=\left\{c_{A, x}, A \subseteq S, x \in A\right\}$ this locality axiom is our $J$-decisiveness axiom for the lattices $\mathcal{S}$ and $\mathcal{C}$. Aizerman and Aleskerov define also a neutrality and monotonicity axiom with respect to the choice functions $c_{A, x}$. For the lattice $\mathcal{S}$ (or $\mathcal{C}$ ) the combination of their two axioms is equivalent to our neutrality monotonicity axiom (see [21]).

Then for these two lattices we have the same axioms and for instance our Theorem 7 for choice functions satisfying the concordance axiom (C) corresponds to case 2 of Theorem 6.10 in [1]. For other lattices of choice functions the axioms are different but can lead to the same classes of consensus functions (in fact these classes are always the class of all federation consensus functions or the restricted classes of oligarchic or co-oligarchic consensus functions). It is for instance the case for our Theorem 8 concerning the choice functions satisfying the outcast axiom $(\mathrm{O})$. We obtain the class of join-projection (co-oligarchic) consensus functions like in Theorem 6.10 quoted above.

Aizerman and Aleskerov's book contains also many results for various strengthenings of their basic axiomatic and/or for other classes of choice functions in particular those which are proper (i.e. such that the choice is never empty) and those obtained by combining the three fundamental axioms $(\mathrm{H}),(\mathrm{C})$ and $(\mathrm{O})$. Recall that one obtains the class of rationalizable (respectively rationalizable by a partial order, or path-independent) choice functions by taking the choice functions satisfying axioms $(\mathrm{H})$ and $(\mathrm{C})$ (respectively axioms $(\mathrm{H}),(\mathrm{C})$ and $(\mathrm{O})$, or axioms $(\mathrm{H})$ and $(\mathrm{O})$ ). These three significant classes of choice functions are not lattices and so one cannot use Theorem 4 or 5 of our paper. However they are join semilattices with the set union as join operation. For instance, the $\cup$-semilattice of path independent choice functions has been studied in [24] (see also [9], [10]). And on the other hand many results of axiomatic and metric latticial consensus theories have been extended to semilattices (see e.g. [11], [12], [13], [14], [18], [19], [21]). Then such results can be applied to the above semilatticial classes of choice functions, modulo the study of the structural properties of such semilattices. Here also one can get again some results of Aizerman and Aleskerov's book or to find different results. Since our aim was more to illustrate the approach using the latticial consensus theory than to enumerate results obtained by this approach we send

\footnotetext{
${ }^{9}$ The metric approach has been considered by people like Mirkin ([20]) but not for choice functions.
} 
back to [27] for cases concerning semilattices of choice functions. Note that work remains to do in this direction since the structure of such semilattices has not always been completely determined. In fact even in the case of the lattices considered here, we don't know all the join-irreducible elements of $\mathcal{O}$ as well as the dependence relations $\beta$ in $\mathcal{C}$ and $\delta$ and $\beta$ in $\mathcal{O}$. We hope to progress on those points in future.

\section{Acknowledgements}

The authors thank two anonymous referee for several useful remarks or corrections on the first version of the paper.

\section{References}

[1] Aizerman MA, Aleskerov F (1995) Theory of choice. North-Holland/ Elsevier, Amsterdam .

[2] Aizerman MA, Malishevski AV (1981) General theory of best variants choice. IEEE Trans. Automatic Control AC - 26(5) pp 1030 - 1041.

[3] Aleskerov FT (1999) Arrovian aggregation models, Kluwer (Theory and decision library. In: Mathematical and Statistical methods 39).

[4] Aleskerov FT, Monjardet B (2002) Utility maximisation, choice and preference. Springer-Verlag, Berlin.

[5] Barthélemy JP, Monjardet B (1981) The median procedure in cluster analysis and social choice theory. Mathematical Social Science 1 pp $235-268$.

[6] Barthélemy JP, Janowitz MF (1991) A formal theory of consensus. SIAM Journal of Discrete Mathematics 4 pp $305-322$.

[7] Birkhoff G (1940) Lattice theory (first ed.) American Mathematical Society Providence.

[8] Davey BA, Priestley HA (1990) Introduction to lattices and order. Cambridge University Press.

[9] Johnson MR, Dean RA (2001) Locally complete path independent choice functions and their lattices. Mathematical social sciences 42 pp 53-87.

[10] Koshevoy GA (1999) Choice functions and abstract convex geometries. Mathematical social sciences 38 pp 35-44.

[11] Leclerc B (1990) Medians and majorities in semi-modular lattices. SIAM Journal of Discrete Mathematics 3 (2) pp 266 - 276.

[12] Leclerc B (1993) Lattice valuations, medians and majorities. Discrete Mathematics 111 pp $345-356$.

[13] Leclerc B (1994) Medians for weight metrics in the covering graphs of semilattices. Discrete Applied Mathematics 49 pp 281-297.

[14] Leclerc B (2003) The median procedure in the semilattice of orders. To appear in: Discrete Applied Mathematics.

[15] Leclerc B, Monjardet B (1995) Latticial theory of consensus. In: Social Choice, Welfare and Ethics, Barnett V, Moulin H, Salles M and Schofield NJ. Cambridge University Press pp 145-159. 
[16] Li J, Boukaabar K (2000) Singular points and an upper bound of medians in upper semi-modular lattices. Order 17 pp $287-299$.

[17] McMorris FR, Powers RC (1995) The median procedure in a formal theory of consensus. SIAM Journal of Discrete Mathematics 14 pp 507-516.

[18] McMorris FR, Mulder HM, Powers RC (2000) The median function on median graphs and semilattices. Discrete Applied Mathematics 101 pp 221-230.

[19] McMorris FR, Mulder HM, Powers RC (2003) The median function on distributive semilattices. To appear in: Discrete Applied Mathematics.

[20] Mirkin BG (1979) Group choice. Wiley New York.

[21] Monjardet B (1990) Arrowian characterizations of latticial federation consensus functions. Mathematical Social Sciences 20(1) pp $51-71$.

[22] Monjardet B (1990) The consequences of Dilworth's work on lattices with unique irreducible decompositions. In: The Dilworth theorems Selected papers of Robert P. Dilworth (eds. Bogart KP, Freese R et Kung J) Birkhaüser, Boston pp 192 - 201.

[23] Monjardet B, Caspard N (1997) On a dependance relation in finite lattices. Discrete Mathematics 165/166 pp 497 - 505 .

[24] Monjardet B, Raderanirina V (2001) The duality between the semilattices of antiexchange closure operators and the path independent choice operators. Mathematical Social Sciences 41 pp 131-150.

[25] Moulin H (1985) Choice functions over a finite set: A summary. Social Choice and Welfare 2(2) pp $147-160$.

[26] Quackenbush RW (1986) Dedekind's problem. Order 2 pp 415-417.

[27] Raderanirina V (2001) Treillis et agrégation de familles de Moore et de fonctions de choix. Thèse de doctorat Université Paris 1. 